\title{
SYNTHESIS AND DOCKING STUDIES OF RENIN INHIBITORS CONTAINING ESTER AND AMIDE DERIVATIVES OF (3S, 4S)-4-AMINO-HYDROXY ACIDS WITH $\mathrm{S}_{3}-\mathrm{S}_{3}$ ' RENIN BINDING SITE
}

\author{
IWONA WINIECKA ${ }^{1 *}$, DOROTA MARSZAŁEK ${ }^{1}$, PAWEŁ JAWORSKI ${ }^{1}$, ANDRZEJ MAZUREK ${ }^{2}$, \\ PAULINA PECAK ${ }^{3}$, KAJETAN WINIECKI ${ }^{3}$ and ARTUR WIERZBOWSKI ${ }^{4}$
}

${ }^{1}$ Department of Drug Chemistry, Medical University of Warsaw, Banacha 1, 02-097 Warsaw, Poland

${ }^{2}$ National Medicines Institute, Chełmska 30/34, 00-727 Warsaw, Poland

${ }^{3} \mathrm{SSG}$ at the Department and Clinic of Psychiatry and Psychotherapy, Medical University of Silesia, Poniatowskiego 15, 40-055 Katowice, Poland

${ }^{4} \mathrm{SSG}$ at the Department of Drug Chemistry, Medical University of Warsaw, Banacha 1, 02-097 Warsaw,

Poland

\begin{abstract}
Seven novel potential renin inhibitors, having a structure based on the peptide fragment 8-13 of human angiotensinogen - a natural substrate for renin, have been designed and synthesized. All peptides were obtained by the carbodiimide method in solution and purified by chromatography on the $\mathrm{SiO}_{2}$ column. In $\mathrm{P}_{1}-\mathrm{P}_{3}$ ' positions, they contain esters or $\mathrm{N}$-alkyl amides of modified (3S, 4S)-4-amino-3-hydroxyacids. The achieved inhibitory activity $\mathrm{IC}_{50}$ was of $10^{-6}-10^{-7} \mathrm{M}$ Inhibitory activity of the compounds was measured in vitro by high-performance liquid chromatography (HPLC) determination of lowering the concentration of substrate in the presence of renin and the potential rennin inhibitor. Their resistance to enzymatic degradation was assayed by the determination of stability against chymotrypsin activity. Their hydrophobicity evaluated as a Log P-value was calculated by a computer method. The theoretical binding affinity of thus obtained inhibitors was elaborated based on docking to the $\mathrm{S}_{3}-\mathrm{S}_{3}$ ' pocket of the renin active site.
\end{abstract}

Keywords: amino acids, hypertension treatment, renin inhibitors, in vitro inhibitory activity determination, docking, pseudodipeptidic unit.

\section{GENERAL}

An active form of renin is formed when the $\mathrm{N}$-terminal 43-amino acid fragment is removed from the zymogen. Renin is responsible for proteolytic cleavage of angiotensinogen to an inactive dodecapeptide, angiotensin I, and stimulates a (Pro)Renin Receptor (PRR) $(1,2)$. Angiotensin I is transformed by convertase enzyme into an active octapeptide angiotensin II (Figure 1). Angiotensin II directly causes blood pressure increase.

Stimulation of PRR by renin leads to proliferation and fibrosis of cardiac and vascular cells $(3,4)$. Pharmacological inhibition of renin bioactivity indirectly prevents angiotensin II from forming and reduces the effects of PRR stimulation by renin. Excessive biosynthesis of angiotensin II and enhanced PRR expression contributes to cardiovascular strain. This mechanism is the basis for the pathogenesis of numerous cardiovascular conditions, such as arterial hypertension, cardiac failure, and ischaemic heart disease. Renin inhibitors, such as Aliskiren (5-7) and neprilysin inhibitors like Sacubitryl or Entresto (8-12), have the most promising therapeutic efficacy amongst the newest family of hypotensives acting by inhibition of enzymatic activity. The low bioavailability of renin inhibitors is the main obstacle

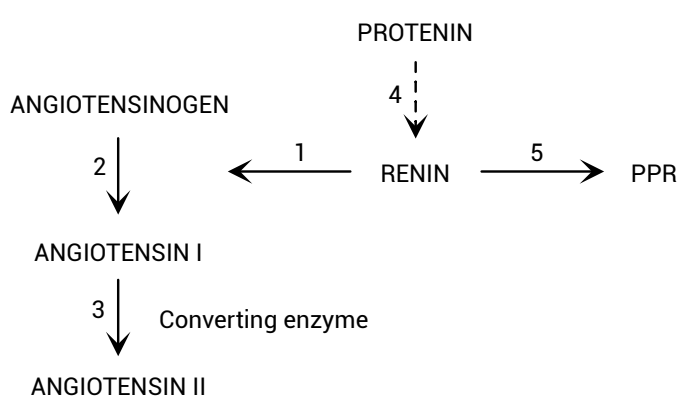

Figure 1. Two-way mechanism of renin action; PRR - (Pro) Renin Receptor; 1, 2, 3- main path of angiotensin II creation; 4 - (Pro) Renin activation; 5 - PRR stimulation by renin. 
in obtaining efficient treatment usability. Improved bioavailability can be achieved by enhancing the stability of sites susceptible to proteolysis and by establishing balanced hydrophobic-hydrophilic properties of the inhibitory molecule. On the other hand, it has to be assured that such modification will not decrease the affinity of the inhibitor to the respective enzyme. The molecule design of presently developed synthetic renin inhibitors is based on the structure of the substrate fragment. The applicable modification of this fragment serves to obtain non-peptide renin inhibitors.

Alterations of the core sites of inhibitory molecules can be carried out twofold. Firstly, by embedding a hydrophobic particle, containing lateral aliphatic, cycloaliphatic, or phenyl substituents, into the molecule. Secondly, by implementing a fragment containing more polar heterocyclic systems, such as piperidine $(13-15)$ or piperazine $(16,17)$ ring.

The hydrophobic part of the renin inhibitor docks at the hydrophobic $\mathrm{S}_{3}\left(\mathrm{~S}_{3}{ }^{\mathrm{sp}}\right)-\mathrm{S}_{1}$ renin pocket (18). This is the key binding mechanism, but hydrophobic interactions of the inhibitor with the $\mathrm{S}_{4}$ and $\mathrm{S}_{2}{ }^{\prime}-\mathrm{S}_{3}$, sites of the enzyme are also important.

The discovery of sub-pockets in $\mathrm{S}_{3}$ and $\mathrm{S}_{1}$ sites, which can interact with hydrophilic and polar groups, such as - $\mathrm{OH},-\mathrm{NH}_{2},-\mathrm{OMe}$, and -OEt groups was of high significance. It enabled new ways of designing renin inhibitors, whose particles can undergo substantial structural changes in $\mathrm{P}_{3}$ and $\mathrm{P}_{1}$ positions.

Facilitated formation of additional hydrogen bonds may lead to increased enzyme-inhibitor interactions, and therefore to enhanced efficacy of potential renin inhibitors. Consequently, designing potential renin inhibitors based on the $\mathrm{P}_{3}-\mathrm{P}_{1}$, fragment of human angiotensinogen, with the necessary modifications, should lead to improved stability and bioavailability of the molecule and enhanced binding affinity to the renin active site (19).

\section{Previous work}

The current study is a followup of our former research activities. For example, the previously synthesized renin inhibitor Boc-Phe(4-OMe) -MeLeu-AHPPA-Ahx-Iaa was taken as a starting point for the design of the inhibitors 12 and $\mathbf{2 5}$ presented here. This compound featured high in vitro inhibitory activity $\mathrm{IC}_{50}=1.05 \times 10^{-9} \mathrm{M}$, which is comparable to the inhibitory activity of Aliskiren $\left(\mathrm{IC}_{50}=0.6 \times 10^{-9} \mathrm{M}\right)$. It was also characterized by a significant hydrophobicity of $\log$ P 7.71 and stability in the chymotrypsin environment (19).

\section{Design of the new inhibitors}

The compound 12, designed in the scope of the current project, differs from the previously (19) synthesized inhibitor Boc-Phe(4-OMe) -MeLeu-AHPPAAhx-Iaa by a pseudopeptide embedded in the $\mathrm{P}_{1}$ $\mathrm{P}_{1}$, position, where AHPPA has been replaced by AEPHPA. Another type of modification to the basic molecule, implemented here, was substituting hydrophobic C-terminal with a more extensively branched one. The compound 25 was created by replacing C-terminal Ahx-Iaa with MeLeu-Iaa in this same basic molecule. The compound $\mathbf{2 0}$ was obtained by implementing this same $\mathrm{C}$-terminal replacement, but applied to the Boc-Phe(4-OMe) -MeLeu-ACPHAAhx-Iaa inhibitor $\left(\mathrm{IC}_{50}=1 \times 10^{-6}\right)$ synthesized earlier (20).

The structure of the new renin inhibitors, described in the present work, was based on the $\mathrm{P}_{3}-\mathrm{P}_{1}$, fragment 8-11 of angiotensinogen (Figure 2) because this part of the substrate molecule plays a significant role in binding to the renin active center. Additionally, the C-terminal fragment corresponding to $\mathrm{P}_{2}$ '- $\mathrm{P}_{3}$ ' position was extended and the effect of this modification on the inhibitory activity of the obtained compounds was investigated. The substituents
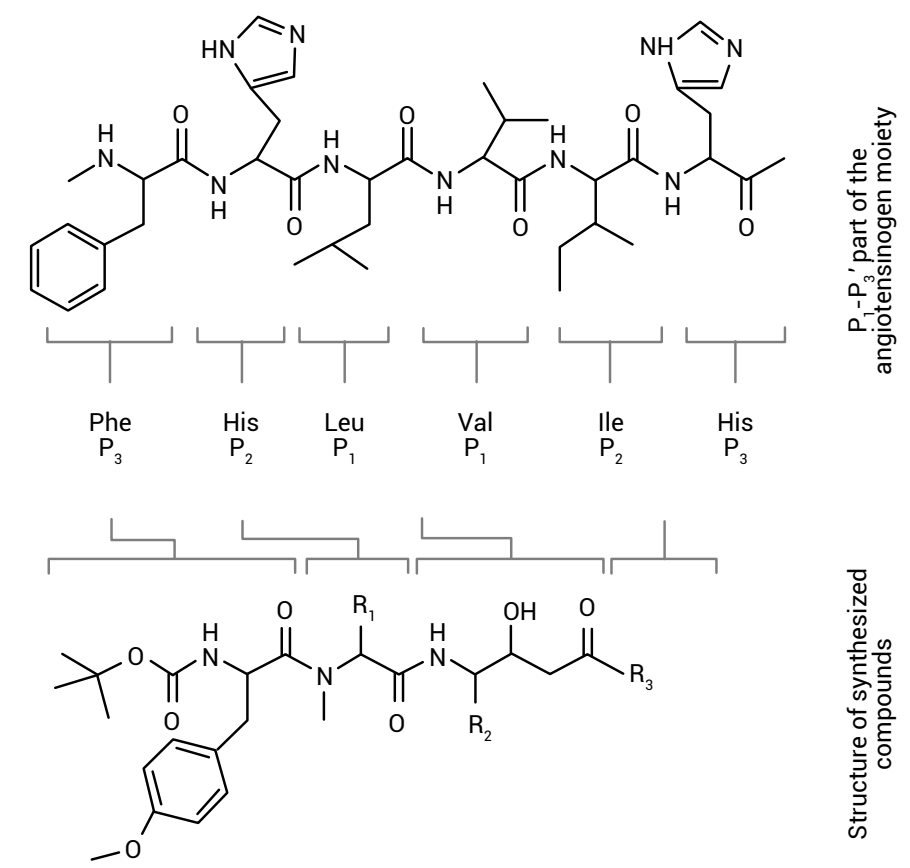

Figure 2. Comparison of general molecule structure of the derived renin inhibitors to the angiotensinogen 8-13fragment (positions $\mathrm{P}_{3}-\mathrm{P}_{3}{ }^{\prime}$ ). 
Table 1. Substituents used for the Synthesized Renin Inhibitors

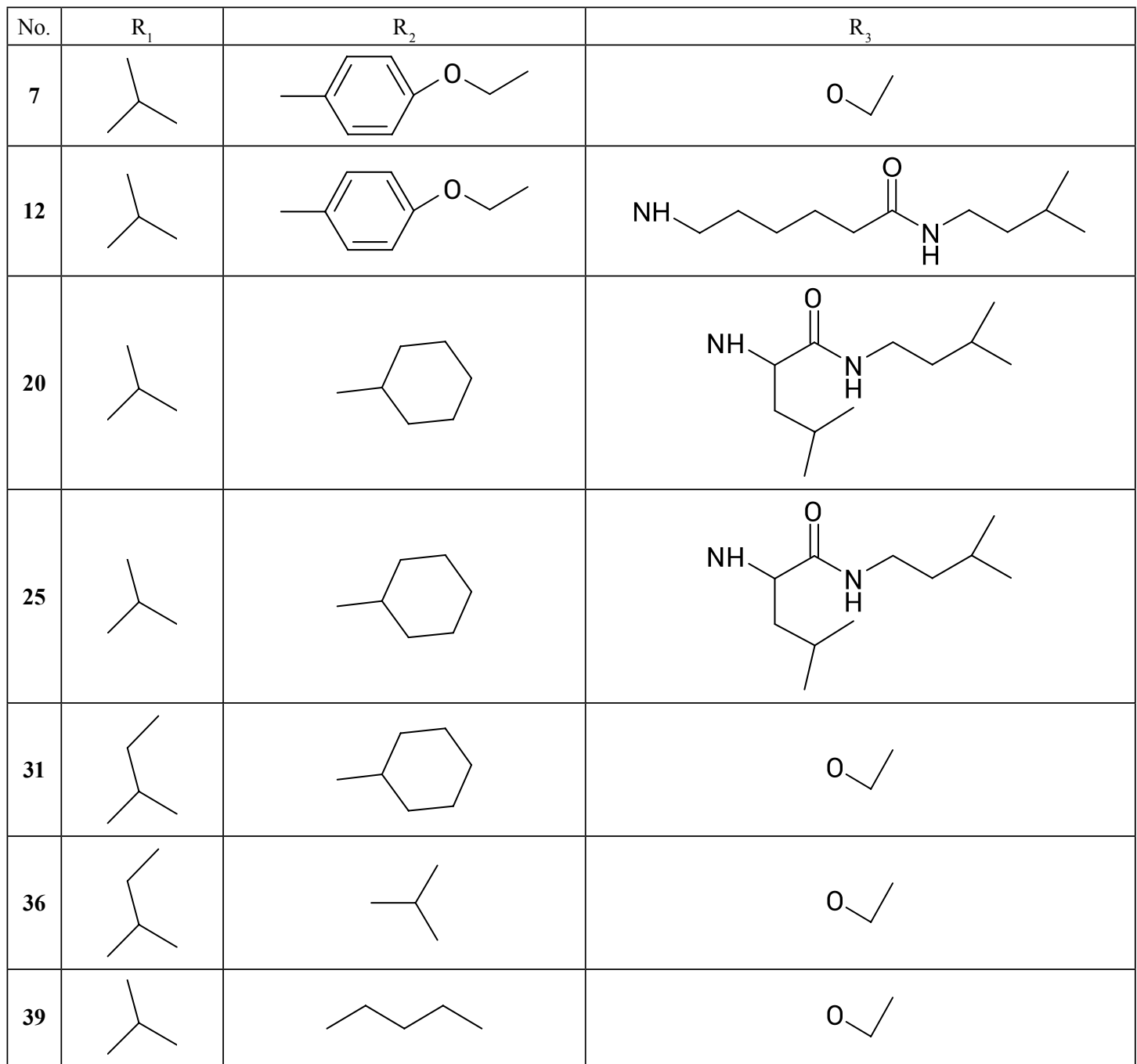

$\mathrm{R}_{1}$ to $\mathrm{R}_{3}$, shown in Figure 2, are for all obtained compounds listed in Table 1.

The $\mathrm{P}_{3}-\mathrm{P}_{2}$ and $\mathrm{P}_{1}-\mathrm{P}_{1}$ ' sites, susceptible to proteolysis (Figure 3), were stabilized by substituting Phe-His and Leu-Val peptide fragments with unnaturally modified dipeptides and pseudodipeptides. In particular, Phe(4-OMe) in combination with various N-methyl amino acids was introduced into the $\mathrm{P}_{3}-\mathrm{P}_{2}$ fragment of the angiotensinogen molecule and non-peptide 4-amino-3-hydroxy acid derivatives were inserted in $\mathrm{P}_{1}-\mathrm{P}_{1}$ ' position (21-23).

Moreover, we made several changes to $\mathrm{N}$ - and C-terminal fragments of inhibitor molecules, considering their influence on the level of interactions with respective $\mathrm{S}_{4}$ and $\mathrm{S}_{2}$ '- $\mathrm{S}_{3}$, hydrophobic pockets of renin (22-24).

Seven new renin inhibitors were synthesized (Table 2). In four of them, C-terminal was extended with ethyl esters of the following amino hydroxyacids: 4-amino-5-(4-ethoxyphenyl)-3-hydroxypentanoic acid (AEPHPA) (compound 7), 4-amino-5-phenyl-3-hydroxypentanoic acid (AHPPA) (compound 31), 4-amino-3-hydroxy6-methylheptanoic acid (Sta) (compound 36), 4-amino-3-hydroxynonanoic acid (AHNA) (compound 39). In the other three compounds, $\mathrm{N}$-isoamyl amino acid amides of the following acids were introduced in the $\mathrm{P}_{2}$ '- $\mathrm{P}_{3}$ ' position: 6-aminohexanoic acid (AhxIaa) associated with AEPHPA (compound 12) and

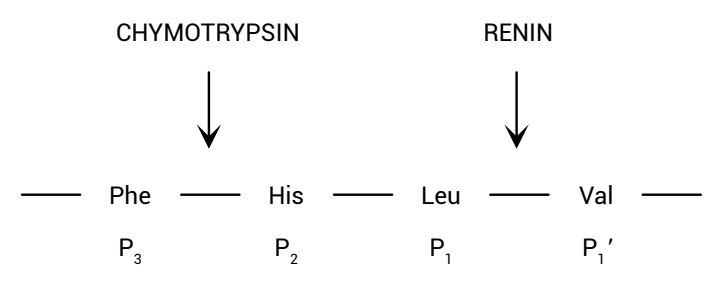

Figure 3. Decomposition scheme of the 8-11 $\left(\mathrm{P}_{3}-\mathrm{P}_{1}{ }^{\prime}\right)$ angiotensinogen fragment by proteolytic enzymes. 
2-(N-methylamino)-4-methylpentanoic acid (MeLeu) associated with 4-amino-5-cyclohexyl-3-hydroxypentanoic acid (ACHPA) (compound 20) and AHPPA (compound 25).

4-Methoxyphenylalanine ( $\mathrm{Phe}(4-\mathrm{OMe})$ ) is present in $\mathrm{P}_{3}$ position of all the synthesized inhibitors, with its amine group substituted with tertbutyloxycarbonyl group (t-Boc) corresponding to $\mathrm{P}_{4}$ position. In $\mathrm{P}_{2}$ position, compounds $7, \mathbf{1 2}, \mathbf{2 0}, \mathbf{2 5}$, and 39 have 2-(N-methylamino)-4-methylpentanoic acid (MeLeu), while compounds 31 and $\mathbf{3 6}$ have 2-(N-methylamino)-4-methylhexanoic acid (MeIle). The studies were aimed at evaluating the bindability of the synthesized potential renin inhibitors, with particular respect to hydrophobic and hydrogen bonds of the compound's molecule to hydrophobic $\mathrm{S}_{3}{ }^{\text {sp }}-\mathrm{P}_{1}$ pocket $(18,19)$. The focus was put on the effect of particular internal interactions between hydrophobic pharmacophores of the inhibitor, resulting from different molecule conformations, which in turn can lead to weakening or enhancing the inhibition of enzymatic renin activity. Replacing the AHPPA amino acid at the position $\mathrm{P}_{1}-\mathrm{P}_{1}$, with AEPHPA or ACHPA shall allow for assessing bindability and affinity differences of the inhibitor fragment to the hydrophobic pocket $\mathrm{S}_{1}$. Conformational properties of the more flexible cyclohexyl ring of ACHPA at the $\mathrm{P}_{1}$ position in comparison to the planar and rigid phenyl ring of AHPPA can provide for a better fit to the hydrophobic pocket of the enzyme active center. The presence of a polar ethoxy substituent in position 4 of the phenyl ring of AEPHPA will probably enable the creation of an additional bound inside the sub-pocket $\mathrm{S}_{1}$. The structural modifications introduced at the $\mathrm{P}_{1}$ position were aiming at the possibly strong interaction between enzyme and the enzyme and inhibitor.

Hydrophobic groups in $\mathrm{P}_{1}, \mathrm{P}_{2}, \mathrm{P}_{3}$, and $\mathrm{P}_{4}$ positions (N-terminal), and $\mathrm{P}_{2}$ '- $\mathrm{P}_{3}$ ' (C-terminal) seem to play a vital role in intramolecular interactions (25-27). It was assumed that possible, intramolecular interactions between $\mathrm{P}_{1}$ side chains and $\mathrm{C}$-terminal

Table 2. Structures of the Synthesized Renin Inhibitors

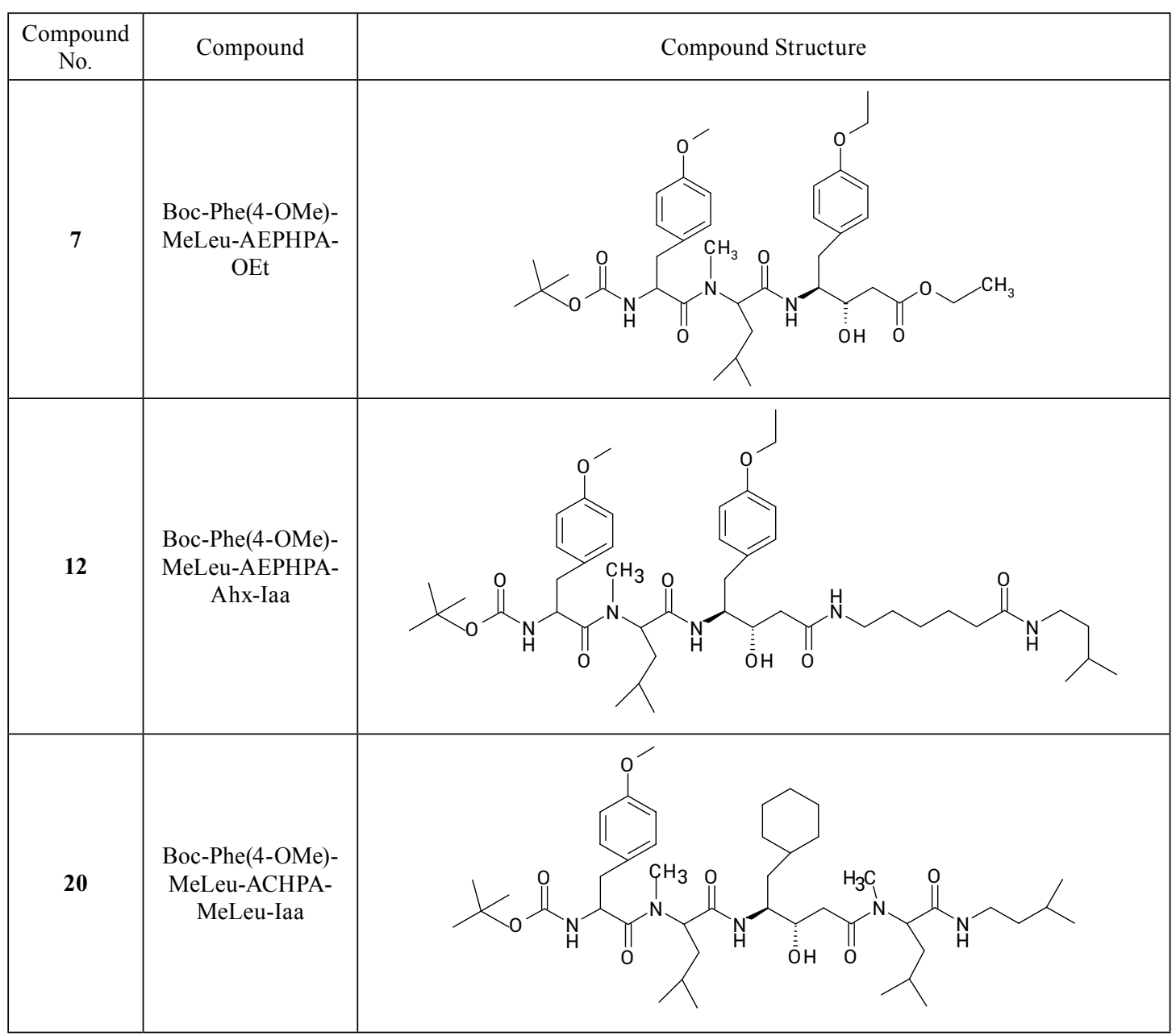


Table 2. Structures of the Synthesized Renin Inhibitors (cont.)

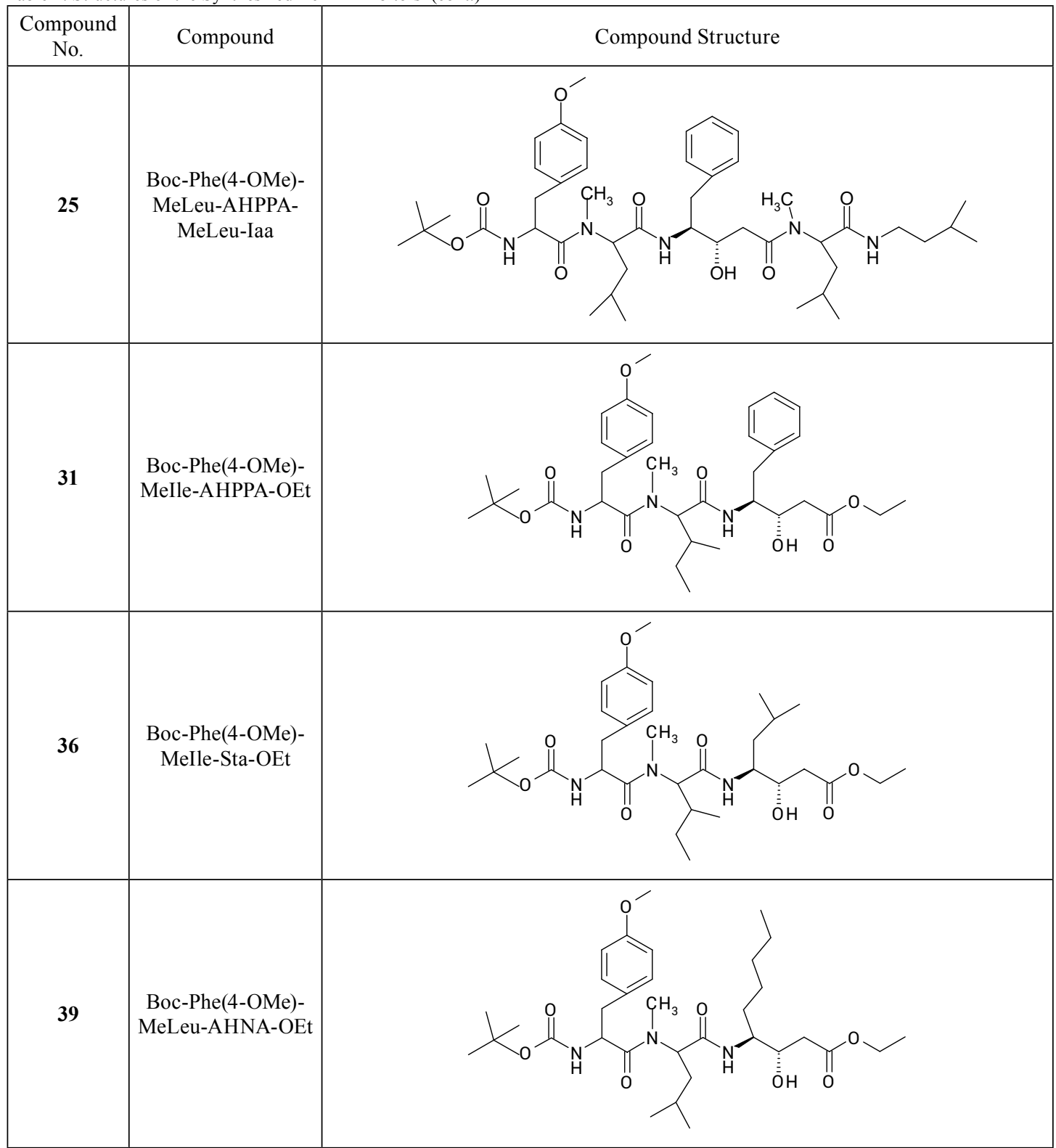

tail $\mathrm{P}_{2}$ '- $\mathrm{P}_{3}$ ' can be particularly interesting. Therefore, the obtained molecules are strongly differentiated in structure, especially in the $\mathrm{P}_{1}$ position and C-terminal tail of the molecule. Comparison between inhibitory concentrations, $\mathrm{IC}_{50}$, and results of studies on docking of the obtained inhibitors to the renin active site can provide answers to the following questions:

- Is an extension of the inhibitor molecule by substituting the $\mathrm{P}_{2}$ '- $\mathrm{P}_{3}$, position with a longer, branched, hydrophobic aliphatic group (N-alkyl amide), as done in compounds $12,20,25$, advantageous when compared to shorter molecules of compounds 7, 31, 36, 39, which are terminated with a polar ester group in $\mathrm{P}_{1}$, position?
- Is bindability of the renin active site influenced by hydrophobic interaction between vicinal molecular fragments, due to the elasticity of chains and ability to obtain various conformations, and if so, to what extent?

For all the obtained compounds, it is probable that intramolecular interactions can take place between substituents in $\mathrm{P}_{1}$ or $\mathrm{P}_{2}$ positions. Another issue is the effect of possible interactions between branched $\mathrm{C}$-terminal ( $\mathrm{N}$-alkylamide fragment), containing isopentyl and isobutyl groups in $\mathrm{P}_{2}$ '- $\mathrm{P}_{3}$ ' positions, and various substituents in $\mathrm{P}_{1}$ position on the inhibitory activity of the compound. 


\section{MATERIALS AND METHODS}

\section{Chemistry}

The structures of inhibitors are presented in Table 2. The inhibitors $7, \mathbf{1 2}, \mathbf{2 0}, \mathbf{2 5}, \mathbf{3 1}, \mathbf{3 6}, \mathbf{3 9}$, as well as their intermediates, were synthesized by fragment condensation, according to schemes presented in Figure 4 to Figure 10.

The applied methods are specified below in the syntheses section. Physicochemical properties of the

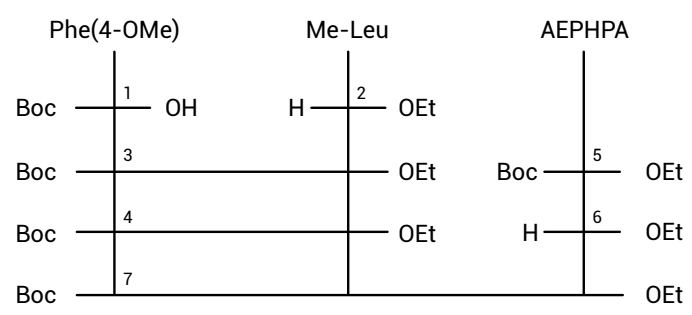

Figure 4. Synthesis of Boc-Phe(4-OMe)-MeLeu-AEPHPA-OEt [N-(t-butoxycarbonyl)-4-methoxyphenylalanyl]-N-methylleucyl(3S,4S)-4-amino-5-(4-ethoxyphenyl)-3-hydroxypentanoic-acid ethyl ester (compound 7).

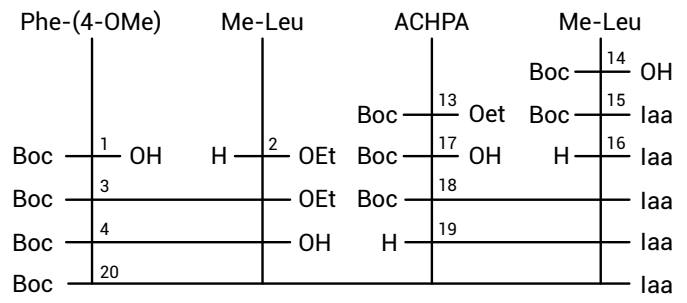

Figure 6. Synthesis of Boc-Phe(4-OMe)-MeLeu-ACHPA-

MeLeu-Iaa [N-(t-butoxycarbonyl)-4-methoxyphenylalanyl]-Nmethylleucyl-(3S,4S)-4-amino-5-cyclohexyl-3-hydroxypentanoyl$\mathrm{N}$-methylleucineisoamyloamide (compound 20).

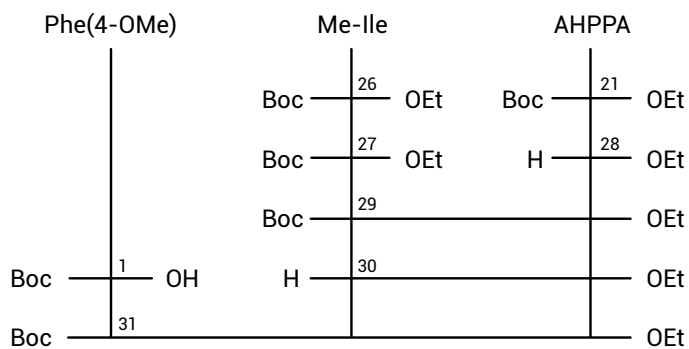

Figure 8. Synthesis of Boc-Phe(4-OMe)-MeIle-AHPPA-OEt [N-(t-butoxycarbonyl)-4-methoxyphenylalanyl]-

Nmethylisoleucyl-(3S,4S)-4-amino-5-phenyl-3-

hydroxypentanoic-acid ethyl ester (compound 31).

Figure 10. Synthesis of Boc-Phe(4-OMe)-MeLeu-AHNA-OEt [N-(t-butoxycarbonyl)-4-methoxyphenylalanyl]-Nmethylleucyl(3S,4S)-4-amino-3-hydroxynonanoic-acid ethyl ester (compound 39) inhibitors, as well as newly synthesized intermediates $3,4,6,10,18,19,23,24,28,29,30,33,34,35$, and 38, are presented in Table 3 and Table 4. The numbers of final compounds in these tables and in the text below are written in bold.

Reagents Boc-Phe(4-OMe)-OH, Boc-N-MeLeu-OH, Boc-N-Me-Ilu-OH, porcine kidney renin, and $\mathrm{N}$-acetyl renin substrate tetradecapeptide were acquired from recognized vendors, listed in the materials section below. The AEPHPA, AHPPA, ACHPA,

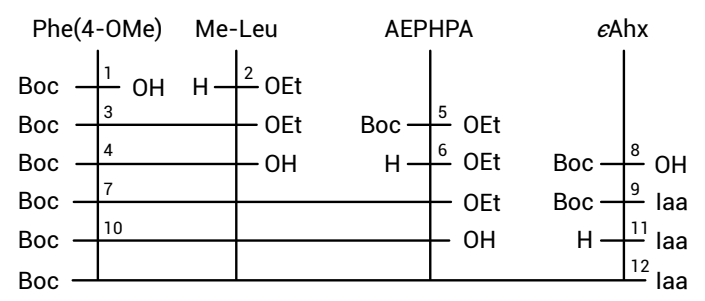

Figure 5. Synthesis of Boc-Phe(4-OMe)-MeLeu-AEPHPA-Ahx-Iaa [N-(t-butoxycarbonyl)-4-methoxyphenylalanyl]-N-methylleucyl(3S,4S)-4-amino-5-(4-ethoxyphenyl)-3-hydroxypentanoylaminohexanoicacid isoamylamide (compound 12).

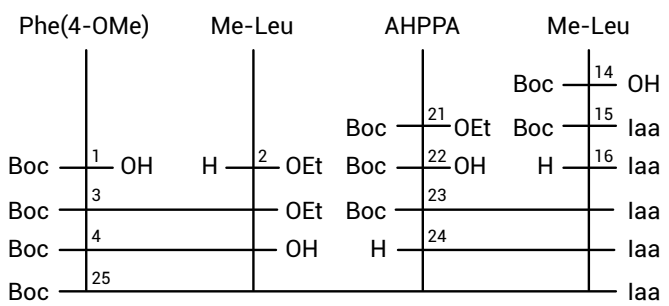

Figure 7. Synthesis of Boc-Phe(4-OMe)-MeLeu-AHPPAMeLeu-Iaa [N-(t-butoxycarbonyl)-4-methoxyphenylalanyl]-Nmethylleucyl-(3S,4S)-4-amino-5-phenyl -3-hydroxypentanoyl-Nmethylleucineisoamyloamide (compound 25).

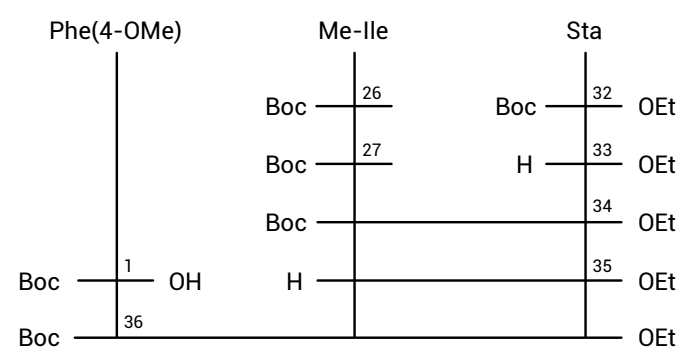

Figure 9. Synthesis of Boc-Phe(4-OMe)-MeIle-Sta-OEt [N-(t-butoxycarbonyl)-4-methoxyphenylalanyl]-

Nmethylisoleucyl-(3S,4S)-4-amino-3-hydroxyheptanoic-acid ethyl ester (compound 36).

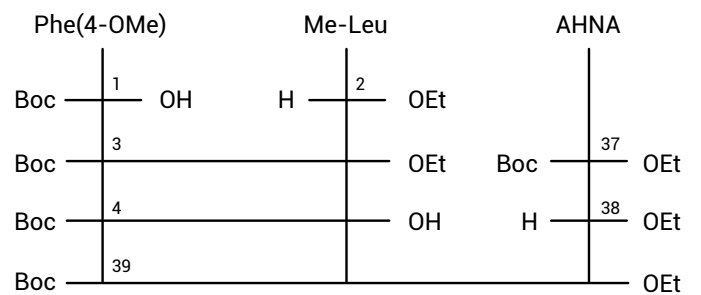


Table 3. Physicochemical and analytical properties of the synthesized compounds.

\begin{tabular}{|c|c|c|c|c|c|c|c|}
\hline No. & Compound & $\begin{array}{l}\text { Formula } \\
\text { m.w. }\end{array}$ & $\begin{array}{l}\text { Yield } \\
(\%)\end{array}$ & $\begin{array}{l}\text { M.p. } \\
\left({ }^{\circ} \mathrm{C}\right)\end{array}$ & $(\mathrm{c}, \mathrm{MeOH})$ & $\begin{array}{l}\text { TLC, } \mathrm{R}_{\mathrm{f}} \\
\text { (m.ph.) }\end{array}$ & $\begin{array}{c}\text { HPLC } \\
\text { (\% of purity) }\end{array}$ \\
\hline 3 & $\begin{array}{c}\text { Boc-Phe(4-OMe)-MeLeu- } \\
\text { OMe }\end{array}$ & $\begin{array}{c}\mathrm{C}_{23} \mathrm{H}_{36} \mathrm{~N}_{2} \mathrm{O}_{6} \\
436.56\end{array}$ & 53.64 & Semi-solid & $\begin{array}{l}-42.2 \\
(1.03)\end{array}$ & $\begin{array}{c}0.33 \\
(\mathrm{~B})\end{array}$ & \\
\hline 4 & Boc-Phe(4-OMe)-MeLeu-OH & $\begin{array}{l}\mathrm{C}_{22} \mathrm{H}_{34} \mathrm{~N}_{2} \mathrm{O}_{5} \\
\quad 422.53\end{array}$ & 14.39 & Semi-solid & $\begin{array}{l}-35.00 \\
(1.08)\end{array}$ & $\begin{array}{l}0.11 \\
(\mathrm{~B})\end{array}$ & \\
\hline 5 & Boc-AEPHPA-OEt & $\begin{array}{c}\mathrm{C}_{20} \mathrm{H}_{31} \mathrm{NO}_{6} \\
381.48\end{array}$ & 37.50 & $83-85$ & $\begin{array}{c}-22.00 \\
(1.0)\end{array}$ & $\begin{array}{l}0.18 \\
(\mathrm{~A})\end{array}$ & \\
\hline 6 & $\mathrm{HCl} \times \mathrm{H}-\mathrm{AEPHPA}-\mathrm{OEt}$ & $\begin{array}{c}\mathrm{C}_{15} \mathrm{H}_{24} \mathrm{NO}_{4} \mathrm{Cl} \\
317.80\end{array}$ & 89.80 & Semi-solid & - & $\begin{array}{c}0.44 \\
(\mathrm{~F})\end{array}$ & \\
\hline 7 & $\begin{array}{c}\text { Boc-Phe(4-OMe)-MeLeu- } \\
\text { AEPHPA-OEt }\end{array}$ & $\begin{array}{c}\mathrm{C}_{37} \mathrm{H}_{55} \mathrm{~N}_{3} \mathrm{O}_{9} \\
685.85\end{array}$ & 53.3 & Semi-solid & $\begin{array}{c}-18.00 \\
(0.6)\end{array}$ & $\begin{array}{l}0.86 \\
\text { (B) }\end{array}$ & 99.18 \\
\hline 10 & $\begin{array}{c}\text { Boc-Phe(4-OMe)-MeLeu- } \\
\text { AEPHPA-OH }\end{array}$ & $\begin{array}{c}\mathrm{C}_{35} \mathrm{H}_{51} \mathrm{~N}_{3} \mathrm{O}_{9} \\
661.83\end{array}$ & 81.40 & Semi-solid & $\begin{array}{c}-178.00 \\
(0.8)\end{array}$ & $\begin{array}{c}0.27 \\
(\mathrm{E})\end{array}$ & \\
\hline 12 & $\begin{array}{c}\text { Boc-Phe(4-OMe)-MeLeu- } \\
\text { AEPHPA-Ahx-Iaa }\end{array}$ & $\begin{array}{c}\mathrm{C}_{46} \mathrm{H}_{73} \mathrm{~N}_{5} \mathrm{O}_{9} \\
840.10 \\
\end{array}$ & 81.50 & Semi-solid & $\begin{array}{c}-79.01 \\
(1.7) \\
\end{array}$ & $0.39(\mathrm{C})$ & 99.67 \\
\hline 13 & Boc-ACHPA-OEt & $\begin{array}{c}\mathrm{C}_{18} \mathrm{H}_{33} \mathrm{NO}_{5} \\
343.44 \\
\end{array}$ & 13.27 & Semi-solid & $\begin{array}{r}-34.0 \\
(1.2)\end{array}$ & $0.42(\mathrm{~A})$ & \\
\hline 17 & Boc-ACHPA-OH & $\begin{array}{c}\mathrm{C}_{16} \mathrm{H}_{29} \mathrm{NO}_{5} \\
317.44 \\
\end{array}$ & 65.02 & Semi-solid & $\begin{array}{l}-28 \\
(1.1) \\
\end{array}$ & $0.62(\mathrm{~B})$ & \\
\hline 18 & Boc-ACHPA-MeLeu-Iaa & $\begin{array}{c}\mathrm{C}_{28} \mathrm{H}_{53} \mathrm{~N}_{3} \mathrm{O}_{5} \\
511.76 \\
\end{array}$ & 27.91 & Semi-solid & $\begin{array}{c}-45 \\
(1.04) \\
\end{array}$ & $0.67(\mathrm{~B})$ & \\
\hline 19 & HCl×H-ACHPA-MeLeu-Iaa & $\begin{array}{c}\mathrm{C}_{23} \mathrm{H}_{45} \mathrm{~N}_{3} \mathrm{O}_{3} \mathrm{Cl} \\
447.09 \\
\end{array}$ & 83.33 & Semi-solid & - & $\begin{array}{c}0.38 \\
(\mathrm{~F})\end{array}$ & \\
\hline 20 & $\begin{array}{c}\text { Boc-Phe(4-OMe)-MeLeu- } \\
\text { ACHPA-MeLeu-Iaa }\end{array}$ & $\begin{array}{c}\mathrm{C}_{45} \mathrm{H}_{77} \mathrm{~N}_{5} \mathrm{O}_{8} \\
816.15 \\
\end{array}$ & 93.75 & Semi-solid & $\begin{array}{c}-52 \\
(0.98) \\
\end{array}$ & $0.28(\mathrm{~B})$ & 97.45 \\
\hline 21 & Boc-AHPPA-OEt & $\begin{array}{c}\mathrm{C}_{18} \mathrm{H}_{26} \mathrm{NO}_{5} \\
336.41 \\
\end{array}$ & 35.30 & $84-86$ & $\begin{array}{c}-30.10 \\
(1.0) \\
\end{array}$ & $0.34(\mathrm{~A})$ & \\
\hline 22 & Boc-AHРPA-OH & $\begin{array}{c}\mathrm{C}_{16} \mathrm{H}_{23} \mathrm{NO}_{5} \\
309.37\end{array}$ & 62.35 & Semi-solid & $\begin{array}{c}-24.10 \\
(1.0)\end{array}$ & $0.60(\mathrm{C})$ & \\
\hline 23 & Boc-AHPPA-MeLeu-Iaa & $\begin{array}{c}\mathrm{C}_{28} \mathrm{H}_{47} \mathrm{~N}_{3} \mathrm{O}_{5} \\
505.70 \\
\end{array}$ & 76.24 & Semi-solid & $\begin{array}{c}-58.00 \\
(1.2) \\
\end{array}$ & $0.89(\mathrm{~A})$ & \\
\hline 24 & HCl×H-AHPPA-MeLeu-Iaa & $\begin{array}{c}\mathrm{C}_{23} \mathrm{H}_{40} \mathrm{~N}_{3} \mathrm{O}_{2} \mathrm{Cl} \\
442.10 \\
\end{array}$ & 96.67 & Semi-solid & - & $\begin{array}{c}0.42 \\
(\mathrm{~F})\end{array}$ & \\
\hline 25 & $\begin{array}{c}\text { Boc-Phe(4-OMe)-MeLeu- } \\
\text { AHPPA-MeLeu-Iaa }\end{array}$ & $\begin{array}{c}\mathrm{C}_{45} \mathrm{H}_{71} \mathrm{~N}_{5} \mathrm{O}_{7} \\
798.08 \\
\end{array}$ & 34.8 & Semi-solid & $\begin{array}{c}+69.17 \\
(1.0) \\
\end{array}$ & $0.34(\mathrm{D})$ & 96.98 \\
\hline 28 & $\mathrm{HCl} \times \mathrm{H}-\mathrm{AHPPA}-\mathrm{OEt}$ & $\begin{array}{c}\mathrm{C}_{13} \mathrm{H}_{20} \mathrm{NO}_{3} \mathrm{Cl} \\
272.72\end{array}$ & 93.0 & Semi-solid & - & $\begin{array}{l}0.20 \\
(\mathrm{~F})\end{array}$ & \\
\hline 29 & Boc-MeIle-AHPPA-OEt & $\begin{array}{c}\mathrm{C}_{25} \mathrm{H}_{40} \mathrm{~N}_{2} \mathrm{O}_{6} \\
464.59\end{array}$ & 49 & Semi-solid & $\begin{array}{c}-16.11 \\
(1.2) \\
\end{array}$ & $0.97(\mathrm{~A})$ & \\
\hline 30 & $\mathrm{HCl} \times \mathrm{H}-\mathrm{MeIle}-\mathrm{AHPPA}-\mathrm{OEt}$ & $\begin{array}{c}\mathrm{C}_{20} \mathrm{H}_{33} \mathrm{~N}_{2} \mathrm{O}_{4} \mathrm{Cl} \\
400.94\end{array}$ & 77.8 & Semi-solid & - & $\begin{array}{c}0.21 \\
(\mathrm{~F})\end{array}$ & \\
\hline 31 & $\begin{array}{c}\text { Boc-Phe(4-OMe)-MeIle- } \\
\text { AHPPA-OEt }\end{array}$ & $\begin{array}{c}\mathrm{C}_{35} \mathrm{H}_{51} \mathrm{~N}_{3} \mathrm{O}_{8} \\
641.79 \\
\end{array}$ & 39.5 & Semi-solid & $\begin{array}{c}-26.42 \\
(1.1) \\
\end{array}$ & $\begin{array}{r}0.93 \\
(\mathrm{E}) \\
\end{array}$ & 99.87 \\
\hline 32 & Boc-Sta-OEt & $\begin{array}{c}\mathrm{C}_{15} \mathrm{H}_{29} \mathrm{NO}_{5} \\
303.41\end{array}$ & 20.8 & Semi-solid & $\begin{array}{l}-39.68 \\
(1.06) \\
\end{array}$ & $0.33(\mathrm{~A})$ & \\
\hline 33 & $\mathrm{HCl} \times \mathrm{H}-\mathrm{Sta}-\mathrm{OEt}$ & $\begin{array}{c}\mathrm{C}_{10} \mathrm{H}_{22} \mathrm{NO}_{3} \mathrm{Cl} \\
239.74\end{array}$ & 86.00 & Semi-solid & - & $0.12(\mathrm{~B})$ & \\
\hline 34 & Boc-MeIle-Sta-OEt & $\begin{array}{c}\mathrm{C}_{22} \mathrm{H}_{42} \mathrm{~N}_{2} \mathrm{O}_{6} \\
430.58\end{array}$ & 65 & Semi-solid & $\begin{array}{r}-17.8 \\
(1.1) \\
\end{array}$ & $0.95(\mathrm{~A})$ & \\
\hline 35 & $\mathrm{HCl} \times \mathrm{H}-\mathrm{MeIle}-\mathrm{Sta}-\mathrm{OEt}$ & $\begin{array}{c}\mathrm{C}_{17} \mathrm{H}_{35} \mathrm{~N}_{2} \mathrm{O}_{4} \mathrm{Cl} \\
366.92 \\
\end{array}$ & 90.6 & Semi-solid & - & $\begin{array}{l}0.31 \\
(\mathrm{~F})\end{array}$ & \\
\hline
\end{tabular}


Table 3. Physicochemical and analytical properties of the synthesized compounds (cont.).

\begin{tabular}{|c|c|c|c|c|c|c|c|}
\hline No. & Compound & $\begin{array}{l}\text { Formula } \\
\text { m.w. }\end{array}$ & $\begin{array}{l}\text { Yield } \\
(\%)\end{array}$ & $\begin{array}{l}\text { M.p. } \\
\left({ }^{\circ} \mathrm{C}\right)\end{array}$ & $(\mathrm{c}, \mathrm{MeOH})$ & $\begin{array}{l}\text { TLC, } \mathrm{R}_{\mathrm{f}} \\
(\mathrm{m} . \mathrm{ph} .)^{*}\end{array}$ & $\begin{array}{c}\text { HPLC } \\
\text { (\% of purity) }\end{array}$ \\
\hline 36 & $\begin{array}{c}\text { Boc-Phe(4-OMe)-MeIle- } \\
\text { Sta-OEt }\end{array}$ & $\begin{array}{l}\mathrm{C}_{32} \mathrm{H}_{53} \mathrm{~N}_{3} \mathrm{O}_{8} \\
607.78\end{array}$ & 45 & Semi-solid & $\begin{array}{c}-111.10 \\
(1.1)\end{array}$ & $0.95(\mathrm{~A})$ & 98.96 \\
\hline 37 & Boc-AHNA-OEt & $\begin{array}{l}\mathrm{C}_{16} \mathrm{H}_{32} \mathrm{NO}_{5} \\
317.44\end{array}$ & 25.34 & $52.4-53.6$ & $\begin{array}{l}+4.5 \\
(1.2)\end{array}$ & $0.60(\mathrm{D})$ & \\
\hline 38 & $\mathrm{HCl} \times \mathrm{H}-\mathrm{AHNA}-\mathrm{OEt}$ & $\begin{array}{l}\mathrm{C}_{11} \mathrm{H}_{25} \mathrm{NO}_{3} \mathrm{Cl} \\
253.77\end{array}$ & 90.58 & Semi-solid & - & $\begin{array}{c}0.47 \\
(\mathrm{~F})\end{array}$ & \\
\hline 39 & $\begin{array}{c}\text { Boc-Phe(4-OMe)-MeLeu- } \\
\text { AHNA-OEt }\end{array}$ & $\begin{array}{l}\mathrm{C}_{33} \mathrm{H}_{55} \mathrm{~N}_{3} \mathrm{O}_{6} \\
621.83\end{array}$ & 57.03 & Semi-solid & $\begin{array}{c}-140.62 \\
(1.1)\end{array}$ & $0.65(\mathrm{~A})$ & 96.97 \\
\hline
\end{tabular}

Mobile phase systems (v/v), were: hexane-EtOAc $80: 20$ (A), $\mathrm{CH}_{3} \mathrm{Cl}-\mathrm{MeOH} 90: 10$ (B), BAW (C),

$\mathrm{CH}_{3} \mathrm{Cl}-\mathrm{MeOH} 99: 1$ (D), $\mathrm{CH}_{3} \mathrm{Cl}-\mathrm{MeOH} 95: 5$ (E), BPW (F).

Table 4. ${ }^{1} \mathrm{H}$ NMR spectra of the synthesized compounds

\begin{tabular}{|c|c|c|}
\hline $\begin{array}{l}\text { Compd. } \\
\text { No }\end{array}$ & Solvent & Chemical shifts $\delta$, ppm \\
\hline 3 & $\mathrm{CDCl}_{3}$ & $\begin{array}{c}0.76-1.84\left(\mathrm{~m}, 9 \mathrm{H}, 2 \times \mathrm{CH}_{3} \mathrm{MeLeu}, 2 \mathrm{HC}_{\beta} \mathrm{MeLeu}, \mathrm{HC}_{\gamma} \mathrm{MeLeu}\right) ; 1.429\left(\mathrm{~s}, 9 \mathrm{H}, \mathrm{C}_{4} \mathrm{H}_{9}\right) ; 2.80-3.24(9 \mathrm{~m} \\
\left.3 \mathrm{H}, \mathrm{N}-\mathrm{CH}_{3}\right) ; 3.24-3.48\left(\mathrm{~m}, 3 \mathrm{H}, \mathrm{OCH}_{3} \text { ester }\right) ; 3.78\left(\mathrm{~s}, 3 \mathrm{H}, \mathrm{OCH}_{3}\right) ; 4.51(\mathrm{~s}, 1 \mathrm{H}, \mathrm{HC} \alpha \mathrm{MeLeu}) \\
4.99\left(\mathrm{~m}, 1 \mathrm{H}, \mathrm{HC}_{\alpha} \mathrm{Phe}(4-\mathrm{OMe})\right) ; 6.82,7.11\left(\mathrm{dd}, 4 \mathrm{H}, \mathrm{C}_{6} \mathrm{H}_{4}\right) ; 7.26(\mathrm{~s}, 1 \mathrm{H}, \mathrm{NH})\end{array}$ \\
\hline 4 & $\mathrm{CDCl}_{3}$ & $\begin{array}{l}0.80-1.84\left(\mathrm{~m}, 8 \mathrm{H}, 2 \times \mathrm{CH}_{3} \mathrm{MeLeu}, 2 \mathrm{HC}_{\beta} \mathrm{MeLeu}\right) ; 1.40\left(\mathrm{~s}, 9 \mathrm{H}, \mathrm{C}_{4} \mathrm{H}_{9}\right) ; 2.30-2.40\left(\mathrm{~m}, \mathrm{HC}_{\gamma} \mathrm{MeLeu}\right) \\
2.82(\mathrm{~s}, 1 \mathrm{H}, \mathrm{OH}) ; 2.90-3.65\left(\mathrm{~m}, 5 \mathrm{H}, \mathrm{N}_{-} \mathrm{CH}_{3}, \mathrm{HC}_{\beta} \mathrm{Phe}(4-\mathrm{OMe})\right) ; 3.79\left(\mathrm{~s}, 3 \mathrm{H}, \mathrm{OCH}_{3}\right) ; 4.55(\mathrm{~d}, 1 \mathrm{H}, \\
\left.\mathrm{HC}_{\alpha} \mathrm{MeLeu}\right) ; 4.93\left(\mathrm{~d}, 1 \mathrm{H}, \mathrm{HC}_{\alpha} \mathrm{Phe}(4-\mathrm{OMe})\right) ; 6.68,7.10\left(\mathrm{dd}, 4 \mathrm{H}, \mathrm{C}_{6} \mathrm{H}_{4}\right) ; 7.26(\mathrm{~s}, 1 \mathrm{H}, \mathrm{NH}) .\end{array}$ \\
\hline 5 & $\mathrm{CDCl}_{3}$ & $\begin{array}{c}1.25\left(\mathrm{t}, 3 \mathrm{H}, \mathrm{CH}_{3} \text { ester }\right) ; 1.39\left(\mathrm{t}, 3 \mathrm{H}, \mathrm{CH}_{3} \text { eter }\right) ; 1.41\left(\mathrm{~s}, 9 \mathrm{H}, \mathrm{C}_{4} \mathrm{H}_{9}\right) ; 2.33-2.62\left(\mathrm{~m}, 2 \mathrm{H}, 2 \mathrm{HC}_{2}\right) \\
2.84\left(\mathrm{~d}, 2 \mathrm{H}, 2 \mathrm{HC}_{5}\right) ; 3.43(\mathrm{~s}, 1 \mathrm{H}, \mathrm{OH}) ; 3.66\left(\mathrm{q}, 1 \mathrm{H}, \mathrm{HC}_{4}\right) ; 3.95-4.04\left(\mathrm{~m}, 3 \mathrm{H}, \mathrm{OCH}_{2} \text { ester, } 1 \mathrm{HC}_{3}\right) \\
\quad 4.13\left(\mathrm{q}, 2 \mathrm{H}, \mathrm{OCH}_{2} \text { eter }\right) ; 4.91(\mathrm{~d}, 1 \mathrm{H}, \mathrm{NH}) ; 6.82\left(\mathrm{~d}, 2 \mathrm{H}, \mathrm{C}_{6} \mathrm{H}_{4}\right) ; 7.15\left(\mathrm{~d}, 2 \mathrm{H}_{6} \mathrm{C}_{6} \mathrm{H}_{4}\right)\end{array}$ \\
\hline 7 & $\mathrm{CDCl}_{3}$ & $\begin{array}{c}\left.\text { 0.80-0.94 (m, } 6 \mathrm{H}, 2 \times \mathrm{CH}_{3} \mathrm{MeLeu}\right) ; 1.12-1.30\left(\mathrm{~m}, 3 \mathrm{H}, \mathrm{CH}_{3} \text { ester }\right) ; 1.34-1.50\left(\mathrm{~m}, 3 \mathrm{H}, \mathrm{CH}_{3} \text { eter }\right) ; \\
1.38\left(\mathrm{~s}, 9 \mathrm{H}, \mathrm{C}_{4} \mathrm{H}_{9}\right) ; 1.50-1.93\left(\mathrm{~m}, 3 \mathrm{H}, 2 \mathrm{HC}_{\beta} \mathrm{MeLeu}, \mathrm{HC}_{\gamma} \mathrm{MeLeu}\right) ; 2.32-3.07\left(\mathrm{~m}, 6 \mathrm{H}, 2 \mathrm{HC}_{2}, 2 \mathrm{HC}_{5},\right. \\
\left.2 \mathrm{HC}_{\beta} \mathrm{Phe}(4-\mathrm{OMe})\right) ; 2.35(\mathrm{~s}, 1 \mathrm{H}, \mathrm{OH}) ; 2.59\left(\mathrm{~s}, 3 \mathrm{H}, \mathrm{N}-\mathrm{CH}_{3}\right) ; 3.77\left(\mathrm{~d}, 3 \mathrm{H}, \mathrm{OCH}_{3}\right) ; 3.90-4.20(\mathrm{~m}, 6 \mathrm{H}, \\
\left.\mathrm{OCH}_{2} \text { ester, } \mathrm{OCH}_{2} \text { eter, HC3, HC4); 4.60-4.78 (m, } 1 \mathrm{H}, \mathrm{HC}_{\alpha} \mathrm{MeLeu}\right) ; 5.07-5.24\left(\mathrm{~m}, 1 \mathrm{H}, \mathrm{HC} \mathrm{Phe}_{\alpha}(4-\right. \\
\left.\mathrm{OMe}^{\mathrm{O}}\right) ; 6.08(\mathrm{~d}, 2 \mathrm{H}, \mathrm{NHAEPHPA}, \mathrm{NHPhe}(4-\mathrm{OMe})) ; 6.73-6.90\left(\mathrm{~m}, 4 \mathrm{H}, \mathrm{C}_{6} \mathrm{H}_{4}\right) ; 7.01-7.17(\mathrm{~m}, 4 \mathrm{H}, \\
\left.\mathrm{C}_{6} \mathrm{H}_{4}\right) .\end{array}$ \\
\hline 10 & $\mathrm{CDCl}_{3}$ & $\begin{array}{c}\left.\text { 0.80-0.94 (m, } 6 \mathrm{H}, 2 \times \mathrm{CH}_{3} \mathrm{MeLeu}\right) ; 1.24-1.47\left(\mathrm{~m}, 3 \mathrm{H}, \mathrm{CH}_{3} \mathrm{eter}\right) ; 1.37\left(\mathrm{~s}, 9 \mathrm{H}, \mathrm{C}_{4} \mathrm{H}_{9}\right) ; 1.50-1.69(\mathrm{~m}, \\
\left.3 \mathrm{H}, 2 \mathrm{HC}_{\beta} \mathrm{MeLeu}, \mathrm{HC}_{\gamma} \mathrm{MeLeu}\right) ; 2.30-3.06\left(6 \mathrm{H}, 2 \mathrm{HC}_{2}, 2 \mathrm{HC}_{5}, 2 \mathrm{HC}_{\beta} \mathrm{Phe}(4-\mathrm{OMe})\right) ; 2.37(\mathrm{~s}, 1 \mathrm{H}, \mathrm{OH}) ; \\
2.73\left(\mathrm{~s}, 3 \mathrm{H}, \mathrm{N}-\mathrm{CH}_{3}\right) ; 3.73\left(\mathrm{~s}, 3 \mathrm{H}, \mathrm{OCH}_{3}\right) ; 3.78\left(\mathrm{q}, 2 \mathrm{H}, \mathrm{OCH}_{2} \mathrm{eter}\right) ; 4.08-4.25\left(\mathrm{~m}_{2}, 2 \mathrm{H}, \mathrm{HC}_{3}, \mathrm{HC}_{4}\right) ; \\
4.70\left(\mathrm{q}, 1 \mathrm{H}, \mathrm{HC}_{\alpha} \mathrm{MeLeu}\right) ; 5.14\left(\mathrm{t}, 1 \mathrm{H}, \mathrm{HC}_{\alpha} \mathrm{Phe}(4-\mathrm{OMe})\right) ; 6.70,6.81\left(\mathrm{dd}, 4 \mathrm{H}, \mathrm{C}_{6} \mathrm{H}_{4}\right) ; 7.06,7.12(\mathrm{dd}, \\
\left.4 \mathrm{H}, \mathrm{C}_{6} \mathrm{H}_{4}\right) .\end{array}$ \\
\hline 12 & $\mathrm{CDCl}_{3}$ & 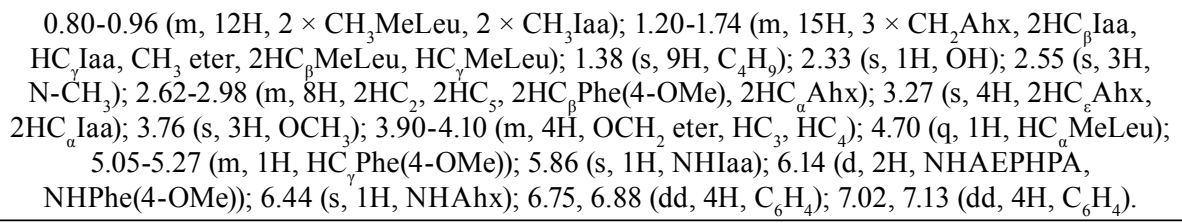 \\
\hline 13 & $\mathrm{CDCl}_{3}$ & $\begin{array}{c}0.78-1.88\left(\mathrm{~m}, 13 \mathrm{H}, \mathrm{C}_{6} \mathrm{H}_{11}, 2 \mathrm{HC}_{5}\right) ; 1.27\left(\mathrm{t}, J=9 \mathrm{~Hz}, 3 \mathrm{H}, \mathrm{CH}_{3}\right) ; 1.44\left(\mathrm{~s}, 9 \mathrm{H}, \mathrm{C}_{4} \mathrm{H}_{9}\right) ; 2.40-2.62 \\
\left(\mathrm{~m}, 2 \mathrm{H}, 2 \mathrm{HC}_{2}\right) ; 3.64\left(\mathrm{q}, J=7 \mathrm{~Hz}, 1 \mathrm{H}, \mathrm{HC}_{4}\right) ; 4.00\left(\mathrm{~d}, J=8 \mathrm{~Hz}, 1 \mathrm{H}, \mathrm{HC}_{3}\right) ; 4.17(\mathrm{q}, J=8 \mathrm{~Hz}, 2 \mathrm{H} \\
\left.\mathrm{OCH}_{2} \text { ester }\right) ; 4.96(\mathrm{~d}, J=12 \mathrm{~Hz}, 1 \mathrm{H}, \mathrm{NH})\end{array}$ \\
\hline 17 & $\mathrm{CDCl}_{3}$ & $\begin{array}{c}0.78-1.88\left(\mathrm{~m}, 13 \mathrm{H}, \mathrm{C}_{6} \mathrm{H}_{11}, 2 \mathrm{HC}_{5}\right) ; 1.44\left(\mathrm{~s}, 9 \mathrm{H}, \mathrm{C}_{4} \mathrm{H}_{9}\right) ; 2.40-2.66\left(\mathrm{~m}, 2 \mathrm{H}, 2 \mathrm{HC}_{2}\right) ; 3.65(\mathrm{q}, J=7 \mathrm{~Hz}, \\
\left.1 \mathrm{H}, \mathrm{HC}_{4}\right) ; 4.00\left(\mathrm{~d}, J=8 \mathrm{~Hz}, 1 \mathrm{H}, \mathrm{HC}_{3}\right) ; 4.82(\mathrm{~d}, J=12 \mathrm{~Hz}, 1 \mathrm{H}, \mathrm{NH}) .\end{array}$ \\
\hline 18 & $\mathrm{CDCl}_{3}$ & 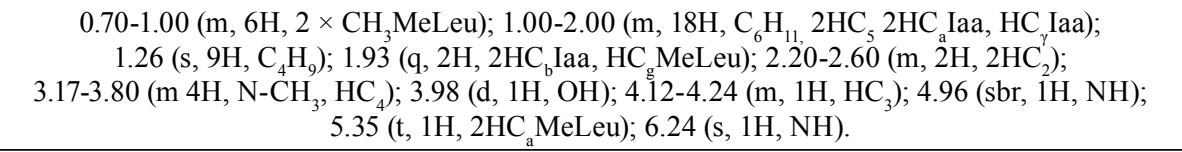 \\
\hline 20 & $\mathrm{CDCl}_{3}$ & $\begin{array}{c}0.78-1.01\left(\mathrm{~m}, 12 \mathrm{H}, 4 \times \mathrm{CH}_{3} \mathrm{MeLeu}\right) ; 1.06-1.50\left(\mathrm{~m}, 13 \mathrm{H}, 2 \mathrm{HC}_{5}, \mathrm{C}_{6} \mathrm{H}_{11}\right) ; 1.42\left(\mathrm{~s}, 9 \mathrm{H}, \mathrm{C}_{4} \mathrm{H}_{9}\right) ; 1.50-1.76 \\
\left(\mathrm{~m}, 6 \mathrm{H}, 2 \times 2 \mathrm{HC}_{\mathrm{b}} \mathrm{MeLeu}, 2 \times \mathrm{HC}_{\mathrm{g}} \mathrm{MeLeu}\right) ; 1.93\left(\mathrm{q}, 2 \mathrm{H}, 2 \mathrm{HC}_{\mathrm{b}} \mathrm{Iaa}\right) ; 2.70\left(\mathrm{~s}, 2 \mathrm{H}, 2 \mathrm{HC}_{2}\right) ; 2.81(\mathrm{~s}, 2 \mathrm{H}, \\
2 \mathrm{HC}_{\beta} \mathrm{Phe}(4-\mathrm{OMe}) ; 2.84-3.36\left(\mathrm{~m}, 6 \mathrm{H}, 2 \times \mathrm{N}_{2} \mathrm{CH}_{3}\right) ; 3.40-3.62\left(\mathrm{~m}, 2 \mathrm{H}, \mathrm{HC}_{3}\right) ; 3.68(\mathrm{~s}, 1 \mathrm{H}, \mathrm{OH}) ; \\
3.71(\mathrm{~s}, 1 \mathrm{H}, \mathrm{NH}) ; 3.79\left(\mathrm{~s}, 3 \mathrm{H}, \mathrm{OCH}_{3}\right) ; 4.74-4.86(\mathrm{~m}, 1 \mathrm{H}, \mathrm{NH}) ; 5.03\left(\mathrm{q}, 1 \mathrm{H}, \mathrm{HC}_{\alpha} \mathrm{Phe}(4-\mathrm{OMe})\right) ; \\
\quad 5.18-5.40\left(\mathrm{~m}, 2 \mathrm{H}, 2 \mathrm{HC}_{\mathrm{a}} \mathrm{MeLeu}\right) ; 5.52(\mathrm{t}, 1 \mathrm{H}, \mathrm{NH}) ; 6.78-7.17\left(\mathrm{~m}, 4 \mathrm{H}, \mathrm{C}_{6} \mathrm{H}_{4}, 1 \mathrm{H}, \mathrm{NH}\right) .\end{array}$ \\
\hline
\end{tabular}


Table 4. 'H NMR spectra of the synthesized compounds (cont.).

\begin{tabular}{|c|c|c|}
\hline \begin{tabular}{|c} 
Compd. \\
No
\end{tabular} & Solvent & Chemical shifts $\delta$, ppm \\
\hline 21 & $\mathrm{CDCl}_{3}$ & $\begin{array}{c}1.28\left(\mathrm{t}, 3 \mathrm{H}, \mathrm{CH}_{3} \text { ester); } 1.37\left(\mathrm{~s}, 9 \mathrm{H}, \mathrm{C}_{4} \mathrm{H}_{9}\right) ; 2.42-2.71\left(\mathrm{~m}, 2 \mathrm{H}, 2 \mathrm{HC}_{5}\right) ; 3.55-4.30\left(\mathrm{~m}, 2 \mathrm{H}, \mathrm{HC}_{3}, \mathrm{HC}_{4}\right) ;\right. \\
3.90\left(\mathrm{~d}, J=6 \mathrm{~Hz}, 2 \mathrm{H}, 2 \mathrm{HC}_{2}\right) ; 4.15\left(\mathrm{q}, J=9 \mathrm{~Hz}, 2 \mathrm{H}, \mathrm{OCH}_{2} \text { ester); } 4.60(\mathrm{~d}, J=8 \mathrm{~Hz}, 1 \mathrm{H}, \mathrm{NH}) ;\right. \\
7.15-7.31\left(\mathrm{~m}, 5 \mathrm{H}, \mathrm{C}_{6} \mathrm{H}_{5}\right)\end{array}$ \\
\hline 22 & $\mathrm{CDCl}_{3}$ & $\begin{array}{l}1.29\left(\mathrm{t}, J=7.0 \mathrm{~Hz}, 3 \mathrm{H}, \mathrm{CH}_{3} \text { ester); } 1.56-1.64\left(\mathrm{~m}, 5 \mathrm{H}, 2 \times \mathrm{CH}_{2}, 1 \times \mathrm{CH}\right) ; 2.48-2.57\left(\mathrm{~m}, 2 \mathrm{H}, \mathrm{CH}_{2}\right) ;\right. \\
3.15-3.25\left(\mathrm{~m}, 2 \mathrm{H}, \mathrm{CH}_{2}\right) ; 3.57(\mathrm{sbr}, 1 \mathrm{H}, \mathrm{CH}) ; 3.98(\mathrm{sbr}, 1 \mathrm{H}, \mathrm{OH}) ; 4.26\left(\mathrm{q}, J=12 \mathrm{~Hz}, 2 \mathrm{H}, \mathrm{OCH}_{2}\right) ; \\
\quad 4.83(\mathrm{~d}, J=9 \mathrm{~Hz}, 1 \mathrm{H}, \mathrm{NH}) ; 5.01(\mathrm{sbr}, 1 \mathrm{H}, \mathrm{NH}) ; 5.19\left(\mathrm{~s}, 2 \mathrm{H}, \mathrm{CH}_{2}\right) ; 7.51\left(\mathrm{~s}, 5 \mathrm{H}, \mathrm{C}_{6} \mathrm{H}_{5}\right) .\end{array}$ \\
\hline 23 & $\mathrm{CDCl}_{3}$ & $\begin{array}{c}1.02-1.19\left(\mathrm{~m}, 6 \mathrm{H}, 2 \times \mathrm{CH}_{3} \mathrm{MeLeu}\right) ; 1.22\left(\mathrm{t}, J=4.0 \mathrm{~Hz}, 3 \mathrm{H}, \mathrm{CH}_{3} \text { ester }\right) ; 1.28-1.49\left(\mathrm{~m}, 1 \mathrm{H}, \mathrm{CH}_{2}\right) ; \\
1.55\left(\mathrm{~s}, 9 \mathrm{H}, \mathrm{C}_{4} \mathrm{H}_{9}\right) ; 1.58-1.84\left(\mathrm{~m}, 7 \mathrm{H}, 2 \mathrm{HC}_{\mathrm{b}} \mathrm{MeLeu}, \mathrm{HC}_{\mathrm{g}} \mathrm{MeLeu}, 2 \mathrm{HC}_{5}, 2 \mathrm{HC}_{6}\right) ; 1.93(\mathrm{dbr}, J=6 \mathrm{~Hz}, \\
\left.2 \mathrm{H}, 2 \mathrm{HC}_{\mathrm{a}} \mathrm{MeLeu}\right) ; 2.75\left(\mathrm{t}, J=4.0 \mathrm{~Hz}, 2 \mathrm{H}, \mathrm{CH}_{2}\right) ; 3.22\left(\mathrm{~d}, J=4 \mathrm{~Hz}, 3 \mathrm{H}, \mathrm{N}-\mathrm{CH}_{3}\right) ; 3.48(\mathrm{~s} \mathrm{br}, 1 \mathrm{H}, \\
\left.\mathrm{CH}_{2}\right) ; 3.83(\mathrm{~s}, 1 \mathrm{H}, \mathrm{OH}) ; 4.15\left(\mathrm{q}, J=8 \mathrm{~Hz}, 2 \mathrm{H}, \mathrm{OCH}_{2} \text { ester }\right) ; 4.96(\mathrm{~s} \mathrm{br}, 1 \mathrm{H}, \mathrm{NH}) ; 5.09\left(\mathrm{~s}, 2 \mathrm{H}, \mathrm{CH}_{2}\right) ; \\
7.26(\mathrm{~s}, 1 \mathrm{H}, \mathrm{NH}) ; 7.34\left(\mathrm{~s}, 5 \mathrm{H}, \mathrm{C}_{6} \mathrm{H}_{5}\right) .\end{array}$ \\
\hline 25 & $\mathrm{CDCl}_{3}$ & $\begin{array}{c}1.16-1.38\left(\mathrm{~m}, 8 \mathrm{H}, 2 \mathrm{HC}_{\mathrm{a}} \mathrm{Iaa}, \mathrm{HC}_{\mathrm{g}} \text { Iaa, } 2 \times \mathrm{CH}_{2} \mathrm{Ahx}, \mathrm{HC}_{3}\right) ; 1.42\left(\mathrm{~s}, 9 \mathrm{H}, \mathrm{C}_{4} \mathrm{H}_{9}\right) ; 1.52-1.86(\mathrm{~m}, 6 \mathrm{H}, \\
\left.2 \times \mathrm{CH}_{2}, 2 \mathrm{HC}_{\mathrm{b}} \mathrm{Iaa}\right) ; 1.86-2.00\left(\mathrm{~m}, 4 \mathrm{H}, 2 \times \mathrm{CH}_{2} \mathrm{Ahx}\right) ; 3.11\left(\mathrm{~d}, J=9.0 \mathrm{~Hz}, 2 \mathrm{H}, 2 \mathrm{HC}_{\mathrm{b}} \mathrm{Phe}(4-\mathrm{OMe})\right) ; \\
3.15\left(\mathrm{~d}, J=4.0 \mathrm{~Hz}, 3 \mathrm{H}, \mathrm{N}-\mathrm{CH}_{3}\right) ; 3.40\left(\mathrm{~s}, 1 \mathrm{H}, \mathrm{CH}_{2}\right) ; 3.78\left(\mathrm{~s}, 3 \mathrm{H}, \mathrm{OCH}_{3}\right) ; \\
\left.\text { 4.53(s br, } 1 \mathrm{H}, \mathrm{HC}_{\mathrm{a}} \mathrm{MeLeu}\right) ; 4.96(\mathrm{~d}, J=9.0 \mathrm{~Hz}, 1 \mathrm{H}, \mathrm{NH}) ; 5.09\left(\mathrm{~s}, 2 \mathrm{H}, \mathrm{CH}_{2} \mathrm{Bzl}\right) ; \\
\left.\text { 6.83-7.10(dd, } J=6.5 \mathrm{~Hz}, J=6.5 \mathrm{~Hz}, 4 \mathrm{H}, \mathrm{C}_{6} \mathrm{H}_{4}\right) ; 7.26(\mathrm{sbr}, 1 \mathrm{H}, \mathrm{NH}) ; 7.34\left(\mathrm{~s}, 5 \mathrm{H}, \mathrm{C}_{6} \mathrm{H}_{5}\right)\end{array}$ \\
\hline 29 & $\mathrm{CDCl}_{3}$ & $\begin{array}{c}0.81-0.90\left(\mathrm{~m}, 6 \mathrm{H}, 2 \times \mathrm{CH}_{3} \mathrm{Ile}\right) ; 1.20-1.29\left(\mathrm{~m}, 5 \mathrm{H}, \mathrm{CH}_{3} \text { ester, } \mathrm{CH}_{2} \mathrm{Ile}\right) ; 1.42-1.53\left(\mathrm{~m}, 9 \mathrm{H}, \mathrm{C}_{4} \mathrm{H}_{9}\right) ; \\
2.38-2.58\left(\mathrm{~m}, 2 \mathrm{H}, 2 \mathrm{HC}_{5}\right) ; 2.62\left(\mathrm{~s}, 3 \mathrm{H}, \mathrm{NCH}_{3}\right) ; 2.82-3.02\left(\mathrm{~m}, 2 \mathrm{H}, 2 \mathrm{HC}_{2}\right) ; 4.00-4.20\left(\mathrm{~m}, 2 \mathrm{H}, \mathrm{OCH}_{2} \text { ester }\right) ; \\
\text { 6.1-6.18 (sbr, } 1 \mathrm{H}, \mathrm{NH}) ; 6.58-6.70(\mathrm{~d}, \mathrm{~J}=9 \mathrm{~Hz}, 1 \mathrm{H}, \mathrm{NH}) ; 7.15-7.38\left(\mathrm{~m}, 5 \mathrm{H}, \mathrm{C}_{6} \mathrm{H}_{5}\right) .\end{array}$ \\
\hline 31 & $\mathrm{CDCl}_{3}$ & $\begin{array}{l}\left.\text { 0.78-1.00 (m, } 6 \mathrm{H}, 2 \times \mathrm{CH}_{3} \mathrm{Ile}\right) ; 1.20-1.58\left(\mathrm{~m}, 16 \mathrm{H}, \mathrm{C}_{4} \mathrm{H}_{9}, \mathrm{CH}_{3} \text { ester, } \mathrm{CH}_{2} \text { Ile, } 2 \mathrm{HC} \text { Ile }\right) ; 1.80-2.00 \\
\left(\mathrm{~m}, 2 \mathrm{H}, 2 \mathrm{HC}_{5}\right) ; 2.60\left(\mathrm{~s}, 3 \mathrm{H}, \mathrm{NCH}_{3}\right) ; 2.78-3.12(\mathrm{~m}, 2 \mathrm{H}, 2 \mathrm{HCPhe}) ; 3.65-3.80\left(\mathrm{~m}, 3 \mathrm{H}, \mathrm{OCH}_{3}\right) ; \\
4.00-4.20\left(\mathrm{~m}, 2 \mathrm{H}, \mathrm{OCH}_{2} \text { ester }\right) ; 5.00(\mathrm{~d}, J=9.3 \mathrm{~Hz}, 1 \mathrm{H}, \mathrm{NH}) ; 6.78-7.22\left(\mathrm{~m}, 9 \mathrm{H}, \mathrm{C}_{6} \mathrm{H}_{5}, \mathrm{C}_{6} \mathrm{H}_{4}\right) .\end{array}$ \\
\hline 32 & $\mathrm{CDCl}_{3}$ & $\begin{array}{c}0.95\left(\mathrm{q}, 6 \mathrm{H}, 2 \times \mathrm{CH}_{3} \mathrm{Sta}\right) ; 1.24-1.28\left(\mathrm{sbr}, 3 \mathrm{H}, \mathrm{CH}_{3} \text { ester }\right) ; 1.46\left(\mathrm{~s}, 9 \mathrm{H}, \mathrm{C}_{4} \mathrm{H}_{9}\right) ; 1.52-1.74\left(\mathrm{~m}, 3 \mathrm{H}, \mathrm{HC}_{6},\right. \\
\left.2 \mathrm{HC}_{5}\right) ; 2.48-2.70\left(\mathrm{~m}, 2 \mathrm{H}, 2 \mathrm{HC}_{2}\right) ; 3.67\left(\mathrm{~s}, 1 \mathrm{H}, \mathrm{HC}_{4}\right) ; 4.013\left(\mathrm{~s}, 1 \mathrm{H}, \mathrm{HC}_{3}\right) ; 4.20\left(\mathrm{q}, 2 \mathrm{H}, \mathrm{OCH}_{2} \text { ester }\right) ; \\
5.00-5.20(\mathrm{~m}, 1 \mathrm{H}, \mathrm{NH}) .\end{array}$ \\
\hline 34 & $\mathrm{CDCl}_{3}$ & $\begin{array}{c}0.82-0.99\left(\mathrm{~m}, 12 \mathrm{H}, 2 \times \mathrm{CH}_{3} \mathrm{Sta}, 2 \times \mathrm{CH}_{3} \mathrm{Ile}\right) ; 1.20-1.30\left(\mathrm{~m}, 3 \mathrm{H}, \mathrm{CH}_{3} \text { ester }\right) ; 1.42-1.52(\mathrm{sbr}, 9 \mathrm{H} \\
\left.\mathrm{C}_{4} \mathrm{H}_{9}\right) ; 1.92-2.16\left(\mathrm{~m}, 3 \mathrm{H}, \mathrm{HC}_{6}, 2 \mathrm{HC}_{5}\right) ; 2.70-2.90\left(\mathrm{~d}, J=9 \mathrm{~Hz}, 2 \mathrm{HC}_{2}\right) ; 3.60-3.80\left(\mathrm{~m}, 3 \mathrm{H}, \mathrm{N}-\mathrm{CH}_{3}\right) ; \\
3.00-4.25\left(\mathrm{~m}, 4 \mathrm{H}, \mathrm{HC}_{3}, \mathrm{HC}_{4}, \mathrm{OCH}_{2} \text { ester }\right) ; 5.00-5.20(\mathrm{~m}, 1 \mathrm{H}, \mathrm{NH}) ; 6.10-6.30(\mathrm{~m}, 1 \mathrm{H}, \mathrm{NH}) .\end{array}$ \\
\hline 36 & $\mathrm{CDCl}_{3}$ & $\begin{array}{c}0.85-1.00\left(\mathrm{~m}, 12 \mathrm{H}, 2 \times \mathrm{CH}_{3} \mathrm{Sta}, 2 \times \mathrm{CH}_{3} \mathrm{Ile}\right) ; 1.19-1.25\left(\mathrm{~m}, 3 \mathrm{H}, \mathrm{CH}_{3} \text { ester }\right) ; 1.41(\mathrm{~s}, 9 \mathrm{H}, \\
\left.\mathrm{C}_{4} \mathrm{H}_{9}\right) ; 1.59\left(\mathrm{~s}, 3 \mathrm{H}, \mathrm{HC}_{6}, 2 \mathrm{HC}_{5}\right) ; 2.74-3.10\left(\mathrm{~m}, 2 \mathrm{H}, \mathrm{CH}_{2} \mathrm{Phe}\right) ; 3.69-3.80\left(\mathrm{~m}, 8 \mathrm{H}, \mathrm{OCH}_{3}, \mathrm{~N}_{-} \mathrm{CH}_{3},\right. \\
\left.\mathrm{OCH}_{2} \text { ester }\right) ; 4.50-4.60\left(\mathrm{~m}, 1 \mathrm{H}, \mathrm{HC}_{2} \mathrm{Ile}\right) ; 6.80-6.86\left(\mathrm{~d}, J=7 \mathrm{~Hz}, 2 \mathrm{H}, \mathrm{C}_{6} \mathrm{H}_{4}\right) ; 7.00-7.03 \\
\left(\mathrm{~d}, J=7.7 \mathrm{~Hz}, 2 \mathrm{H}, \mathrm{C}_{6} \mathrm{H}_{4}\right) .\end{array}$ \\
\hline 37 & $\mathrm{CDCl}_{3}$ & $\begin{array}{c}\left.\text { 0.84-0.92 (m, } 3 \mathrm{H}, \mathrm{CH}_{3}\right) ; 1.20-1.36\left(\mathrm{~m}, 7 \mathrm{H}, \mathrm{CH}_{3} \text { ester, } 2 \times \mathrm{CH}_{2}\right) ; 1.443\left(\mathrm{~s}, 9 \mathrm{H}, \mathrm{C}_{4} \mathrm{H}_{9}\right) ; 2.40-2.60(\mathrm{~m}, \\
\left.2 \mathrm{H}, \mathrm{CH}_{2}\right) ; 3.40-3.55(\mathrm{~m}, 1 \mathrm{H}, \mathrm{NH}) ; 4.0-4.1(\mathrm{~d}, J=2 \mathrm{~Hz}, 1 \mathrm{H}, \mathrm{CH}) ; 4.18\left(\mathrm{q}, 2 \mathrm{H}, \mathrm{OCH}_{2}\right) ; \\
4.7(\mathrm{~d}, J=2 \mathrm{~Hz}, 1 \mathrm{H}, \mathrm{NH}) .\end{array}$ \\
\hline 39 & $\mathrm{CDCl}_{3}$ & $\begin{array}{c}\left.\text { 0.76-1.00 (m, 3H, } \mathrm{CH}_{3} \text { ester }\right) ; 1.10-1.50\left(\mathrm{~m}, 19 \mathrm{H}, 2 \times \mathrm{CH}_{3}, 2 \times \mathrm{CH}_{2}, \mathrm{C}_{4} \mathrm{H}_{9}\right) ; 1.5-2.0\left(\mathrm{~m}, 5 \mathrm{H}, \mathrm{CH}_{2}\right) ; \\
\left.\text { 3.00-3.45(m, } 6 \mathrm{H}, \mathrm{OCH}_{3}, \mathrm{~N}-\mathrm{CH}_{3}\right) ; 3.70(\mathrm{~s}, 1 \mathrm{H}, \mathrm{NH}) ; 4.20-4.40\left(\mathrm{~m}, 2 \mathrm{H}, \mathrm{OCH}_{2}\right) ; 5.30(\mathrm{~m}, 1 \mathrm{H}, \mathrm{NH}) ; \\
7.50-7.80\left(\mathrm{~m}, 4 \mathrm{H}, \mathrm{C}_{6} \mathrm{H}_{4}\right) .\end{array}$ \\
\hline
\end{tabular}

Sta, AHNA, and intermediate compounds 5, 13, 21, 32, 37 were synthesized according to the Maibaum protocol (28). Solvents were of analytical purity. Tetrahydrofuran (THF) was distilled from $\mathrm{Na} /$ benzophenone under $\mathrm{N}_{2}$. Dichloromethane and dimethylformamide (DMF) were dried over $4 \AA$ molecular sieves. The peptides were synthesized by the N,Ndicyclohexylcarbodiimide/1-hydroxybenzotriazole (DCCl/HOBt) method of fragment condensation in solution. Column chromatography (CC) on silica gel (Merck, grade 230 to 400 mesh) was used to separate and purify all synthesized compounds. TLC was carried out on $0.25 \mathrm{~mm}$ thickness silica gel plates (Merck, silica gel $60 \mathrm{~F}_{254}$. The solvent systems used in TLC and $\mathrm{CC}$ were $\mathrm{CHCl}_{3} / \mathrm{MeOH}$ and hexane/EtOAc in various ratios. The spots were visualized with $0.3 \%$ ninhydrin in EtOH/AcOH (97: 3, v/v). Böetius apparatus was used to determine melting points. Bruker DM $300 \mathrm{MHz}$ Avance $300 \mathrm{WB}$ spectrometer was applied to record ${ }^{1} \mathrm{H}$ NMR. Chemical shifts were measured relative to tetramethylsilane (TMS) as $\delta$ units (ppm). Optical rotations were measured at the Na-D line with the use of AP-300 (Atago) polarimeter in a $5 \mathrm{~cm}$ polarimeter cell. HPLC analyses of purity and activity of synthesized inhibitors were performed on a Shimadzu apparatus equipped with an LC-10AT pump, UV-Vis SPD-10A detector, and Chromax 2010 recorder. The peaks were recorded at $213 \mathrm{~nm}$. The separation was carried out in the reverse phase system (Wide Pore $\mathrm{C} 8$, Symmetry C18) with various mobile phases. 


\section{Syntheses \\ Materials}

All standard protected Boc-aminoacid derivatives and angiotensinogen were obtained from Bachem AG (Switzerland). $\alpha$-Chymotrypsin, dicyclohexylcarbodiimid, and isopropyl-magnesium chloride solution were obtained from Sigma-Aldrich Chemie GmbH (Germany). Acetonitrile isocratic grade and methanol for liquid chromatography were acquired at LiChrosolv/Merck Millipore (Germany). Renin was purchased at Cayman Chemical Company (USA).

\section{Synthesis of pseudopeptides}

A two-step method was applied. In the first step, pseudopeptide Boc-ketoesters are obtained. Solution (A), containing $10 \mathrm{mmol}$ of Boc-aminoacid (Boc-Cha-OH, Boc-Phe-OEt, Boc-Phe(4-OEt)-OH, Boc-Leu-OH, Boc-Hep-OH) in $30 \mathrm{~mL}$ of THF was activated with $12 \mathrm{mmol}$ of $\mathrm{N}, \mathrm{N}$-carbonyldiimidazole (DCl). Solution (B), containing $15 \mathrm{mmol}$ of malonic acid monoethyl ester in $30 \mathrm{~mL}$ of THF was activated with $30 \mathrm{mmol}$ of isopropyl magnesium chloride. Both solutions were mixed in $-20^{\circ} \mathrm{C}$ and agitated. Boc-pseudopeptide esters were obtained in the second step. $10 \mathrm{mmol}$ of pseudopeptide Boc-ketoester was dissolved in $100 \mathrm{~mL}$ of THF-MeOH mixture (98: 2) and then reduced with $2.5 \mathrm{mmol}^{\circ} \mathrm{NaBH}_{4}$ in $-78^{\circ} \mathrm{C}(28)$. In this way, a mixture of diastereomers was obtained.

The identity of diastereoisomers was confirmed in the analysis of a specific rotation. According to the literature, diastereoisomers with S,S conformation of the hydroxyethylated pseudodipeptides exhibit higher specific rotation when compared with the R,S diastereoisomers (29-31), which is as follows: (S,S) Boc-AEPHPA-OEt $=-22.00,(\mathrm{R}, \mathrm{S})$ Boc-AEPHPA-OEt $=-5.00$; (S,S) Boc-ACHPA$\mathrm{OEt}=-34.70$, (R,S) Boc-ACHPA-OEt $=-20.04$; $(\mathrm{S}, \mathrm{S})$ Boc-AHPPA-OEt $=-33.0,(\mathrm{R}, \mathrm{S})$ Boc-AHPPA$\mathrm{OEt}=-13.40$; (S,S) Boc-Sta-OEt $=-39.60$, (R,S) Boc$\mathrm{Sta}-\mathrm{OEt}=-10.40$; $(\mathrm{S}, \mathrm{S})$ Boc-AHNA-OEt $=+24.39$, $(\mathrm{R}, \mathrm{S})$ Boc-AHNA-OEt $=+4.50$.

\section{Introduction of the N-tert-Boc-Group}

This group was introduced in a commonly used manner (28).

\section{Removal of the N-tert-Boc group}

Boc-aminoacid or Boc-peptide $(1 \mathrm{mmol})$ in a solution of $4 \mathrm{M} \mathrm{HCl}$ in dioxane $(3-5 \mathrm{~mL}$ ) was stirred at room temperature for $30 \mathrm{~min}$. The solution was conc. in vacuo, then the residue was evaporated twice with ethyl ether and dried in vacuo (32).

\section{Esterification and hydrolysis}

Boc-AEPHPA-OEt, Boc-AHPPA-OEt, BocACHPA-OEt, Boc-Sta-OEt, and Boc-AHNA-OEt were formed from mono-ethyl malonate used to prepare these compounds (33). Alkaline hydrolysis of the ester group was carried out as described (34).

\section{Coupling reaction with DCCI/HOBt}

The amino acid or peptide ester hydrochloride (1mmol) was dissolved in $\mathrm{CH}_{2} \mathrm{Cl}_{2}(5 \mathrm{~mL})$ and neutralized at $0^{\circ} \mathrm{C}$ with THF $(1 \mathrm{mmol})$. Boc-amino acid or Boc-peptide $(1 \mathrm{mmol})$ and 1-hydroxybenzotriazole (HOBt) $(1.5 \mathrm{mmol})$ were added followed by a solution of N,N'-dicyclohexylcarbodiimide (DCCI) $(1.1 \mathrm{mmol})$ in $\mathrm{CH}_{2} \mathrm{Cl}_{2}(5 \mathrm{~mL})$. The reaction mixture was stirred at $0^{\circ} \mathrm{C}$ for $2-4 \mathrm{~h}$ and left at room temperature overnight. Dicyclohexylurea (DCU) was filtered off and the filtrate was evaporated in vacuo. The residue was dissolved in $\mathrm{CHCl}_{3}$, washed successively with $5 \% \mathrm{HCl}, 5 \% \mathrm{NaHCO}_{3}$, saturated $\mathrm{NaCl}$ solution, dried with anh. $\mathrm{MgSO}_{4}$ and conc. in vacuo. The peptide was purified by silica gel CC to yield the pure product (35).

\section{Biochemical assay \\ Determination of inhibition of renin activity}

Renin inhibiting activity of the synthesized potential inhibitors was determined in vitro. HPLC method was used to determine the concentration of renin substrate $(36,37)$. The renin inhibitory activity was designed in terms of the $\mathrm{IC}_{50}$, which is the molar concentration of the examined inhibitor causing 50\% inhibition of the control renin activity. The inhibition of human renin was determined after its incubation with angiotensinogen and with each of compounds 1-7. The activity of the compounds was tested in the range $10^{-6}-10^{-7} \mathrm{M}$.

\section{Stability in the presence of $\alpha$-chymotrypsin}

$\alpha$-Chymotrypsin was dissolved in a phosphate buffer solution $\mathrm{pH}$ 7.8. The solution was incubated at $37^{\circ} \mathrm{C}$ and the samples were collected at the time points $0,30,60,90$, and $120 \mathrm{~min}$. Then, the samples were isolated from the solution with diethyl ether, evaporated to dryness, dissolved in the mobile phase, and determined by HPLC (37).

\section{Log P-value calculation}

Because of the poor solubility of the compounds in water and in n-octanol the Log $\mathrm{P}$ values were calculated by a computer program. Structures of the compounds were modeled with the use of HyperChem Professional 8.0 software and the semiempirical method MP3 was used for single-point 
calculation. Geometry optimization was performed with the Polak-Ribier method. Calculation of Log P in HyperChem was carried out using atomic parameters derived by Ghose (38).

\section{The molecular docking}

The molecular docking studies were performed using BIOVIA Discovery Studio software (39) in which the Flexible Docking workflow was applied to obtain ligand-protein complexes. This approach allows some receptor flexibility during the docking of flexible ligands. Here, low-energy ligands conformations are docked into the active site of each receptor side-chain conformation. Moreover, a side-chain residues optimization around docked ligand positions was carried out. It should be emphasized that protein flexibility may play an important role in the mechanism of ligand docking and should not be ignored during this kind of study.

\section{RESULTS AND DISCUSSION}

All the obtained renin inhibitors have an inhibitory activity of $\mathrm{IC}_{50}$ between $10^{-6}$ and $10^{-7} \mathrm{M}$ (Table 5). Compounds 25, 36, 39 turned out to be the most active $\left(\mathrm{IC}_{50}\right.$ of $\left.10^{-7} \mathrm{M}\right)$, from which the most prominent blocker was compound 25, i.e. Boc-Phe(4-OMe)MeLeu-AHPPA-MeLeu-Iaa with $\mathrm{IC}_{50}=1.58 \times 10^{-7} \mathrm{M}$. Inhibitor compounds 36 and 39 contain hydrophobic, aliphatic groups in the $\mathrm{P}_{1}$ position, and polar C-terminal tail of an alkyl ester structure. Inhibitor 25 contains AHPPA with a cyclic, aromatic group in position $\mathrm{P}_{1}$ and a branched C-terminal chain in $\mathrm{P}_{2}$ '- $\mathrm{P}_{3}$, position, unlike inhibitor compounds $\mathbf{3 6}$ and $\mathbf{3 9}$.

Compounds $\mathbf{3 6}$ and $\mathbf{3 9}$ have only slightly lower inhibitory activity in comparison to compound $\mathbf{2 5}$. It allows assuming that the absence of hydrophobic interaction between C-terminus and $\mathrm{S}_{2}{ }^{\prime}-\mathrm{S}_{3}$, site is compensated by an elastic aliphatic chain in the $\mathrm{P}_{1}$ position, easily fitting to the $\mathrm{S}_{1}$ hydrophobic pocket.

High hydrophobicity of the C-terminal tail, advantageous -long and branched-chain, and its interaction with $\mathrm{S}_{2}$ '- $\mathrm{S}_{3}$ ' hydrophobic pocket of compound 25 seems to be affinity-determining upon strong binding to renin. Benzene ring in $\mathrm{P}_{1}$ position seems to be of a less significant effect, as a compound of an ester structure 31, containing AHPPA pseudopeptide in $\mathrm{P}_{1}$ position, but devoid of a hydrophobic $\mathrm{C}$-terminal tail has a lower activity $\left(\mathrm{IC}_{50}\right.$ of $\left.0.95 \times 10^{-6} \mathrm{M}\right)$. The above-described considerations have given rise to a conclusion that compounds with a $\mathrm{C}$-terminal tail of ester structure have higher inhibitory activity towards renin when $\mathrm{P}_{1}$ position is substituted with non-branched 39 or branched $\mathbf{3 6}$ aliphatic group. Compounds with C-terminal amide structure specifically branched $\mathrm{N}$-alkyl amides, have higher activity when $\mathrm{P}_{1}$ position contains an unsubstituted aromatic ring 25. Potential inhibitor compounds 25 and 20 differ only by $\mathrm{P}_{1}-\mathrm{P}_{1}$ ' fragment structure (phenyl and cyclohexyl ring) and have an inhibitory activity of $\mathrm{IC}_{50}$ of $1.58 \times 10^{-7}$ and $1.19 \times 10^{-6} \mathrm{M}$, respectively. Inhibitors with an ester structure, with an aliphatic group at $\mathrm{P}_{1}$ position, i.e. compounds 36 and 39, are an order of magnitude more active than inhibitors with ester structure of C-terminal tail containing aromatic ring groups at $\mathrm{P}_{1}$ position 7 and $\mathbf{3 1}$. It can eventually be concluded that the highest inhibitory activity towards renin is exhibited by inhibitors with long, aliphatic, and branched hydrophobic chains at $\mathrm{P}_{1}$ and $\mathrm{P}_{2}{ }^{\prime}-\mathrm{P}_{3}$ ' positions. A minor difference in the structure of the side chains at $\mathrm{P}_{2}$ position of inhibitors 36 and 39, respectively containing sec-butyl and isobutyl groups, seems to have the same minor effect on the activity of these inhibitors due to similar space occupancy of those groups. Our studies revealed that a hydrophobic N-terminal tail is not necessary for obtaining inhibitory activity in synthesized compounds.

Table 5. Stability and activity of the synthesized compounds.

\begin{tabular}{|c|c|c|c|c|}
\hline Compound No. & Compound & $\mathrm{IC}_{50}[\mathrm{M}]$ & $\begin{array}{c}\text { Degree of stability } \\
\text { against chymotrypsin** }\end{array}$ & $*$ Log P \\
\hline $\mathbf{7}$ & Boc-Phe(4-OMe)-MeLeu-AEPHPA-OEt & $1.6 \times 10^{-6}$ & Unstable & 4.38 \\
\hline $\mathbf{1 2}$ & Boc-Phe(4-OMe)-MeLeu-AEPHPA-Ahx-Iaa & $1.3 \times 10^{-6}$ & Stable & 5.08 \\
\hline $\mathbf{2 0}$ & Boc-Phe(4-OMe)-MeLeu-ACHPA-MeLeu-Iaa & $1.19 \times 10^{-6}$ & Stable & 5.94 \\
\hline $\mathbf{2 5}$ & Boc-Phe(4-OMe)-MeLeu-AHPPA-MeLeu-Iaa & $1.58 \times 10^{-7}$ & Unstable & 5.99 \\
\hline $\mathbf{3 1}$ & Boc-Phe(4-OMe)-MeIle-AHPPA-OEt & $0.96 \times 10^{-6}$ & Unstable & 4.37 \\
\hline $\mathbf{3 6}$ & Boc-Phe(4-OMe)-MeIle-Sta-OEt & $1.12 \times 10^{-7}$ & Unstable & 3.88 \\
\hline $\mathbf{3 9}$ & Boc-Phe(4-OMe)-MeLeu-AHNA-OEt & $0.96 \times 10^{-7}$ & Stable & 3.93 \\
\hline
\end{tabular}

${ }^{*}$ Hydrophobicity of the compounds expressed as Log P-value was calculated by a computational method (38).

${ }^{* *}$ Fraction of inhibitor amount remaining after $2 \mathrm{~h}$ incubation time in presence of chymotrypsin at $37^{\circ} \mathrm{C}(36)$. 
t-Boc group removal increases the solubility of compounds, as a polar N-terminal tail is formed. Due to a small difference in inhibitory activity towards renin, as determined in in vitro studies, evaluation of effects on binding inhibitors with renin, hydrophobic interactions between $\mathrm{P}_{2}$ and $\mathrm{P}_{1}$ position-substituted groups, and between groups at $\mathrm{P}_{1}$ and $\mathrm{P}_{2}{ }^{\prime}-\mathrm{P}_{3}$ ' positions is difficult to establish. Mutual interaction between side chains at positions $\mathrm{P}_{2}$ and $\mathrm{P}_{1}$ has probably no significant effect on the bioactivity of inhibitors.

$\mathrm{The} \mathrm{IC}_{50}$ values for compounds of similar structures, differentiated only by C-terminal tail structure, either ester or $\mathrm{N}$-alkyl amide, are similar. This suggests that hydrophobic interactions of branched chains within $\mathrm{P}_{2}$ '- $\mathrm{P}_{3}$ ' and $\mathrm{S}_{2}$ '- $\mathrm{S}_{3}$ ' renin site can be reduced due to steric interactions of $\mathrm{N}$-isopentyl and $\mathrm{N}$-isobutyl chains with hydrophobic groups at $\mathrm{P}_{1}$ position within the inhibitor molecules. This, in turn, allows for a conclusion that hydrogen bonds play a vital role in binding inhibitors to the renin active site.

The results of in vitro activity and stability assessment for the obtained compounds indicate that modifications implemented to obtain compounds $\mathbf{1 2}$, 20 and 25, did not increase their inhibition capability of renin activity. They also did not significantly affect their stability against chymotrypsin (Table 5).

Pre-sampled conformations of each of the eight compounds were docked to the binding site of the renin model, which was developed on the basis of the crystal structure of renin-indole-piperazin inhibitor complexes (PDB: 3OOT) reported by Scheiper et al. (40). Taking into consideration the mode of action of renin inhibitors, it can be assumed that, in the case of compounds regarded here, the following residues play an essential role: Gln13, Val30, Thr77, Asp32, Val120, Asp215, Gly217, Ala218, Ser219, and His287. Hence, the binding pocket was defined within these residues. It should be emphasized that binding to the catalytic aspartate residues is vital for all protease inhibitors. This may lead to the conclusion that potential renin inhibitors should interact with Asp32 and/ or Asp215 residues in the renin's active site (41-43).

Analysis of the docking results, binding orientations, and intermolecular interactions of compounds $7,12,20,25,31,36,38$ with the active site revealed that all studied molecules fit well the active site of renin. A large number of degrees of freedom and, thus, many conformations of studied compounds make the analysis difficult. The most discriminative parameters, which distinctly classify the quality of docking, are number and strength (equivalently length and geometry) of the hydrogen bonds formed between ligand and the corresponding amino acids inside the receptor-binding pocket but also, although to a less degree, intermolecular interactions of other types, e.g. hydrophobic and edge-to-face.

A closer inspection of the obtained results revealed that different binding modes can be distinguished inside the set of studied compounds. Apparently, the orientation of the docked ligand inside the active pocket is the most important factor. Here, at least two scenarios can be delineated - studied compounds can be oriented in the way that either the $\mathrm{P}_{4}$ and $\mathrm{P}_{3}$ fragments or $\mathrm{P}_{2}$, and $\mathrm{P}_{1}-\mathrm{P}_{1}$, fragments are faced to the inside of the renin's active site. The former configuration can be attributed to compound 31 and the latter is characteristic especially for compounds 7, 25, 36, and 39. Compounds 12 and 20 approach the active site mainly with the $\mathrm{P}_{2}$ ' fragment, whereas the $\mathrm{P}_{1}-\mathrm{P}_{1}$ ' fragment is placed rather on the verge of it. Therefore, a hypothesis can be made that the binding mode, affinity, and most likely, also the activity of the studied compounds strongly depends on the structure of the $\mathrm{P}_{2}$, and $\mathrm{P}_{1}-\mathrm{P}_{1}$ ' fragments. It is noteworthy that the $\mathrm{P}_{1}-\mathrm{P}_{1}$, fragment is engaged into the vast majority of interactions between ligands and renin's active site residues.

According to in vitro studies, in which the affinity of analyzed compounds to renin was measured, one can assume that molecules 25, 36, and 39 may be the most potent renin's ligands. Indeed, this hypothesis may be strengthened by docking results as compounds $\mathbf{2 5}, \mathbf{3 6}$, and 39 seem to have the same binding mode in which $\mathrm{P}_{2}$, and $\mathrm{P}_{1}-\mathrm{P}_{1}$, fragments are faced to the inside of the renin's active site. The obtained complexes are stabilized by a vast array of intermolecular interactions such as conventional hydrogen bonds, carbon-hydrogen bonds, or hydrophobic interactions. The latter seems to play a pivotal role as a few characteristic hydrophobic surfaces can be distinguished within the renin's active site. This particular binding mode, characteristic for compounds 7 , 25, 36, 39 (as well as $\mathbf{1 2}$ and $\mathbf{2 0}$, to a certain extent) can be illustrated by interactions of compound $\mathbf{3 9}$ with the renin active site residues (Figure 11).

Molecule 39 fits the renin's active site very well. It is worth noting that the docked ligand is oriented in the way that the AHNA ( $\mathrm{P}_{1}-\mathrm{P}_{1}$, fragment), OEt ( $\mathrm{P}_{2}$ ' fragment), and MeLeu ( $\mathrm{P}_{2}$ fragment) face the center of the binding pocket whereas the Phe(4$\mathrm{OMe})\left(\mathrm{P}_{3}\right.$ fragment) slightly extends beyond an analyzed active site and the Boc group ( $\mathrm{P}_{4}$ fragment) is on the verge of it. Even though there are no conventional hydrogen bonds between the studied molecule and Asp215, this particular amino acid is engaged in carbon-hydrogen bonding interactions via its oxygen atom towards hydrogen atoms of the OEt moiety of the $\mathrm{P}_{2}$ ' fragment $(\sim 2.3 \AA)$. Another carbon-hydrogen 


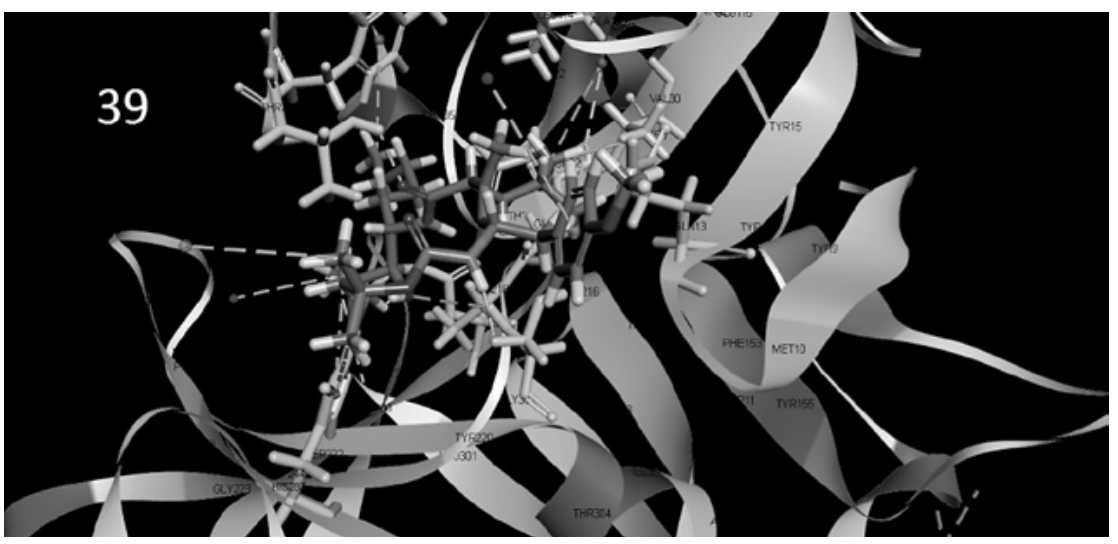

Figure 11. Compound 39 inside the renin's active pocket.

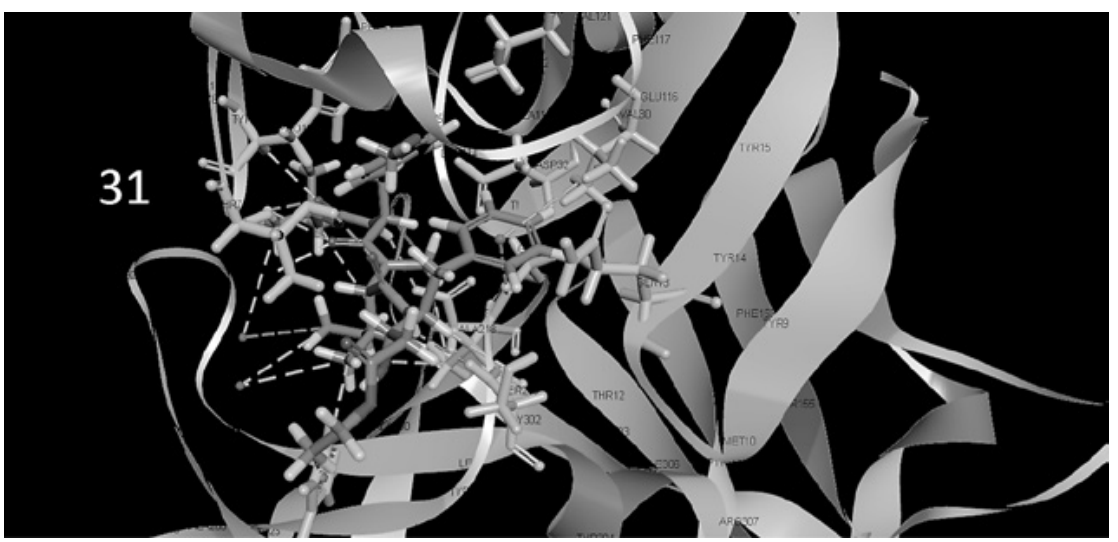

Figure 12. Compound $\mathbf{3 1}$ inside the renin's active pocket.

bonds, yet less important, are formed between $\mathrm{C}=\mathrm{O}$ of the $\mathrm{P}_{3}$ fragment and hydrogen atom of the Ser219 $(\sim 3.0 \AA)$ and between the $\mathrm{C}=\mathrm{O}$ of the $\mathrm{P}_{1}-\mathrm{P}_{1}$, fragment and Ser222 ( 2.4 $\AA$ ). The analyzed complex is stabilized by two hydrogen bonds, which are formed between the -OH group of Thr77 and $\mathrm{C}=\mathrm{O}$ of the $\mathrm{P}_{2}$ fragment $(\sim 2.2 \AA)$ as well as between the -NH group of Ser219 and the oxygen atom of the -OH group of the $\mathrm{P}_{1}-\mathrm{P}_{1}$ ' fragment $(\sim 3.0 \AA)$; the latter one seems to be of less importance. Moreover, hydrophobic interactions can be distinguished between $\mathrm{P}_{2}$ fragment of the molecule 39 and Val30 (alkyl-alkyl), the $\mathrm{P}_{1}-\mathrm{P}_{1}$ ' fragment's side chain and Phe(4-OMe) of Tyr75 (pi-alkyl) and Boc residue of the studied ligand $\left(\mathrm{P}_{4}\right.$, fragment) and both the imidazole ring of His287 (pi-alkyl) and the phenyl ring of the Phe243 (pi-alkyl). Moreover, the $-\mathrm{CH}_{3}$ group of C-terminal fragment of the molecule interacts with Ala300 (alkyl interaction) and with Ile291 (alkyl interaction). Yet other hydrophobic interactions can be distinguished between the $\mathrm{P}_{2}$ fragment of the molecule (MeLeu's$\mathrm{CH}_{3}$ terminal groups) and phenyl ring of Phe117 (pialkyl) and between the phenyl ring of the $\mathrm{P}_{3}$ fragment of the molecule and Leu114 (pi-alkyl).
As already mentioned, compound $\mathbf{3 1}$ represents a different binding mode (Figure 12), in which the docked ligand is oriented in the way that the Boc residue ( $\mathrm{P}_{4}$ fragment), the $\mathrm{Phe}(4-\mathrm{OMe})$ of $\mathrm{P}_{3}$ fragment, and to a much lesser degree, the phenyl group of the AHPPA ( $\mathrm{P}_{1}-\mathrm{P}_{1}$ ' fragment) face the center of the binding pocket and the $\mathrm{P}_{2}$ ' fragment of the studied molecule slightly extends beyond the analyzed active site. This particular complex is stabilized by a few hydrogen bonds, carbon-hydrogen bonds, and some hydrophobic interactions. Here, the hydrogen bond formed between the - $\mathrm{NH}$ - group of the $\mathrm{P}_{3}$ fragment and the oxygen atom of Asp215 ( 2.4 $\AA$ ) should be emphasized. Moreover, a hydrogen bonding interaction can be found between the $-\mathrm{OH}$ group of Thr77 and the $\mathrm{C}=\mathrm{O}$ of the peptide bond of the $\mathrm{P}_{2}$ fragment $(\sim 2.3 \AA)$. Another hydrogen bond can be identified between the $\mathrm{C}=\mathrm{O}$ group of $\mathrm{P}_{3}$ fragment as well as between the $\mathrm{C}=\mathrm{O}$ group of $\mathrm{P}_{4}$ ' fragment of the molecule 31 and -NH- group of $\operatorname{Ser} 76(\sim 2.7 \AA$ and $\sim 2.5 \AA$, respectively). Furthermore, carbon-hydrogen bonds are also formed between the $\mathrm{C}=\mathrm{O}$ of the $\mathrm{P}_{1}-\mathrm{P}_{1}$ ' fragment of the molecule and Tyr75 hydrogen atom $(\sim 2.9 \AA)$, the $\mathrm{C}=\mathrm{O}$ of the $\mathrm{P}_{3}$ fragment and the 
Table. 6. Details of interactions between inhibitors and renin active center

\begin{tabular}{|c|c|c|c|c|}
\hline Ligand No. & Orientation of the docked ligand & Interacting Residues & Category & Type \\
\hline \multirow{13}{*}{39} & \multirow{13}{*}{$\begin{array}{l}\text { Ligand is oriented in the way that the } \\
\text { AHNA }\left(\mathrm{P}_{1}-\mathrm{P}_{1}^{\prime} \text { fragment }\right) \text {, OEt }\left(\mathrm{P}_{2},\right. \\
\text { fragment), and MeLeu }\left(\mathrm{P}_{2} \text { fragment }\right) \\
\text { face the center of the binding pocket } \\
\text { whereas the Phe }(4-\mathrm{OMe})\left(\mathrm{P}_{3} \text { fragment }\right) \\
\text { slightly extends beyond an analyzed } \\
\text { active site and the Boc group } \\
\left(\mathrm{P}_{4} \text { fragment }\right) \text { is on the verge of it. }\end{array}$} & Thr77 & $\begin{array}{c}\text { H-Bond } \\
\sim 2.2 \AA\end{array}$ & Conventional \\
\hline & & Ser219 & $\begin{array}{c}\text { H-Bond } \\
\sim 3.0 \AA\end{array}$ & Conventional \\
\hline & & Asp215 & $\begin{array}{c}\text { H-Bond } \\
\sim 2.3 \AA\end{array}$ & Carbon hydrogen bond \\
\hline & & Ser219 & $\begin{array}{c}\text { H-Bond } \\
\sim 3.0 \AA\end{array}$ & Carbon hydrogen bond \\
\hline & & Ser222 & $\begin{array}{c}\text { H-Bond } \\
\sim 2.4 \AA\end{array}$ & Carbon hydrogen bond \\
\hline & & Val30 & Hydrophobic & Alkyl \\
\hline & & Ala300 & Hydrophobic & Alkyl \\
\hline & & Ile291 & Hydrophobic & Alkyl \\
\hline & & Tyr75 & Hydrophobic & Pi-Alkyl \\
\hline & & His 287 & Hydrophobic & Pi-Alkyl \\
\hline & & Phe243 & Hydrophobic & Pi-Alkyl \\
\hline & & Phe117 & Hydrophobic & Pi-Alkyl \\
\hline & & Leu114 & Hydrophobic & Pi-Alkyl \\
\hline \multirow{13}{*}{31} & \multirow{13}{*}{$\begin{array}{l}\text { Ligand is oriented in the way that the } \\
\text { Boc residue ( } \mathrm{P}_{4} \text { fragment), the Phe }(4- \\
\text { OMe) of } \mathrm{P}_{3} \text { fragment, and to a much } \\
\text { lesser degree, the phenyl group of the } \\
\text { AHPPA ( } \mathrm{P}_{1}-\mathrm{P}_{1} \text { fragment) face the } \\
\text { center of the binding pocket and the } \\
\mathrm{P}_{2} \text { ' fragment of the studied molecule } \\
\text { slightly extends beyond the analyzed } \\
\text { active site. }\end{array}$} & Asp215 & $\begin{array}{c}\text { H-Bond } \\
\sim 2.4 \AA\end{array}$ & Conventional \\
\hline & & Thr77 & $\begin{array}{c}\text { H-Bond } \\
\sim 2.3 \AA\end{array}$ & Conventional \\
\hline & & \multirow{3}{*}{ Ser76 } & $\begin{array}{c}\text { H-Bond } \\
\sim 2.5 \AA\end{array}$ & Conventional \\
\hline & & & $\begin{array}{l}\text { H-Bond } \\
\sim 2.7 \AA \\
\end{array}$ & Conventional \\
\hline & & & $\begin{array}{c}\text { H-Bond } \\
\sim 2.5 \AA \\
\end{array}$ & Carbon hydrogen bond \\
\hline & & Tyr75 & $\begin{array}{c}\text { H-Bond } \\
\sim 2.9 \AA\end{array}$ & Carbon hydrogen bond \\
\hline & & Ser219 & $\begin{array}{c}\text { H-Bond } \\
\sim 2.7 \AA\end{array}$ & Carbon hydrogen bond \\
\hline & & Ala218 & Hydrophobic & Alkyl \\
\hline & & Met289 & Hydrophobic & Alkyl \\
\hline & & Ile291 & Hydrophobic & Alkyl \\
\hline & & Leu213 & Hydrophobic & Alkyl \\
\hline & & Val30 & Hydrophobic & Pi-Alkyl \\
\hline & & His 287 & Hydrophobic & Pi-Alkyl \\
\hline
\end{tabular}

hydrogen atom of Ser76 $(\sim 2.5 \AA)$, and between the -OH group of Ser219 and a hydrogen atom attached to the $\mathrm{C}_{4}$ of the $\mathrm{P}_{1}-\mathrm{P}_{1}$, peptide bond. Additionally, the molecule 31 complex with renin active site is stabilized by hydrophobic interactions, in which the $-\mathrm{CH}_{3}$ group of Ala218 is engaged into alkyl interactions with the $\mathrm{N}-\mathrm{CH}_{3}$ group of the $\mathrm{P}_{2}$ fragment as well as with the $-\mathrm{CH}_{3}$ group attached to $\mathrm{C}_{3}$ of the $\mathrm{P}_{2}$ fragment. Moreover, a pi-alkyl hydrophobic interaction can be distinguished between the
$-\mathrm{CH}_{3}$ group of the $\mathrm{C}$-terminal fragment of the molecule and the imidazole ring of His 287. The same imidazole ring of His287 interacts with molecule 31 via its $-\mathrm{CH}_{3}$ group attached to $\mathrm{C}_{3}$ of the $\mathrm{P}_{2}$ fragment (pi-alkyl). The same fragment of molecule $\mathbf{3 1}\left(\mathrm{C}_{5}\right.$ of the Ile chain) is stabilized by alkyl hydrophobic interactions with Met289 and Ile291. Furthermore, the Boc group of the $\mathrm{P}_{4}$ fragment interacts with the Leu213 and Ile291 residues within the renin's active site via alkyl hydrophobic interactions. Yet other 
hydrophobic interactions (pi-alkyl) can be found between the phenyl ring of the $\mathrm{P}_{1}-\mathrm{P}_{1}$, fragment of the molecule and Val30.

A comprehensive summary of docking results including a description of the most relevant interactions between ligands 31, 39, and the renin active center can be found in Table 6 .

Taking into consideration all the above, it can be assumed that there are a few important features, which determine studied ligands affinity (and most likely activity) towards renin:

1. Binding mode in which $\mathrm{P}_{2}$, and $\mathrm{P}_{1}-\mathrm{P}_{1}$, fragments are faced to the inside of the renin's active site.

2. Structure of the $\mathrm{P}_{2}$, and $\mathrm{P}_{1}-\mathrm{P}_{1}$ ' fragments: a. It seems that aliphatic hydrocarbon chains are preferred (branched or not); they are important in the case of hydrophobic interactions where the flexibility and, hence, the ease of fitting hydrophobic surfaces inside the active site is an asset.

b. The phenyl ring (substituted or not) of AHPPA may trigger some steric hindrances and, therefore, impede the proper emplacement of the ligand in the active site. This observation is quite relative but becomes evident upon the comparison of compounds with AHPPA or ACHPA in their structure with compounds without those fragments or with hydrocarbon chains (branched or not).

3. Branching of $\mathrm{P}_{2}$, and $\mathrm{P}_{1}-\mathrm{P}_{1}$ ' fragments with hydrocarbon chains may improve the ligand's adaptation to the active site's cavity.

\section{CONCLUSIONS}

Compounds without a hydrophobic C-terminal tail, i.e., ester-structure compounds 7, 31, 36, 39 of $\log P=4$, exhibit weaker hydrophobic interactions with respective pockets (Table 5). Compounds $\mathbf{2 0}$ and $\mathbf{2 5}$, as well as compound $\mathbf{1 2}$, have the most prominent hydrophobic interaction capacity due to the high renin binding affinity of $\log P=6$ and $\log P=$ 5 , respectively. Those compounds have a branched C-terminus. Higher hydrophobicity is not a direct estimation of higher in vitro inhibitory activity in each and every case. Compounds $\mathbf{3 6}$ and 39, with an ester structure, and without $\mathrm{P}_{2}$ '- $\mathrm{P}_{3}$ ' fragment, for which hydrophobic constant amounts to $\log \mathrm{P}=3.88$ for compound 36 and $\log \mathrm{P}=3.93$ for compound 39, revealed only slightly decreased inhibitory activity of $\mathrm{IC}_{50}$ of $1.12 \times 10^{-7} \mathrm{M}$ for compound 36 and $0.96 \times$
$10^{-7} \mathrm{M}$ for compound 39 when compared to the most active inhibitor $\mathbf{2 5}$ with a branched aliphatic chain at the $\mathrm{C}$-terminus, of $\log \mathrm{P}=5.99$, and $\mathrm{IC}_{50}=1.58$ $\times 10^{-7} \mathrm{M}$. This suggests a significant contribution of other interactions, particularly hydrogen bonds between the inhibitor molecule and renin active site, which is confirmed by docking studies.

\section{Acknowledgments}

This work was financially supported by the Students Research 'Mini-Grant' FW22/NM1/18 founded by the Deanship of Scientific Research at the Faculty of Pharmacy / Medical University of Warsaw.

\section{Conflicts of interest}

The authors declare no conflict of interest.

\section{REFERENCES}

1. Danser A.H.: Am. J. Hypertens. 28, 1187 (2015).

2. Schroten N.F., Gaillard C.A., van Veldhuisen D.J., Szymanski M.K., Hillege H.L., de Boer R.A.: Heart Fail Rev. 17, 191 (2012).

3. Mahmud H., Candido W.M., van Genne L., Vreeswijk-Baudoin I., Yu H., et al.: PLoS One 9, e89929 (2014).

4. Batenburg W.W., Danser A.H.: Clin. Sci. (Lond) 123, 121 (2012).

5. Vaidyanathan S., Jarugula V., Dieterich H.A., Howard D., Dole W.P.: Clin. Pharmacokinet. 47, 515 (2008).

6. Wood J.M., Maibaum J., Rahuel J. Grütter M.G., Cohen N.C., et al.: Biochem. Biophys. Res. Commun. 308, 698 (2003).

7. Tani S., Kushiro T., Takahashi A., Kawamata H., Ohkubo K., et al.: Intern. Med. 55, 427 (2016).

8. Von Lueder T.G., Atar D., Krum H.: Pharmacol. Ther. 144, 41 (2014).

9. McMurray J.J., Packer M, Desai A.S., Gong J., Lefkowitz M.P., et al.: N. Engl. J. Med. 371, 993 (2014).

10. McMurray J.J., Packer M., Desai A.S., Gong J., Lefkowitz M.P., et al.: Eur. J. Heart Fail. 15, 1062 (2015).

11. Gu J., Noe A., Chandra P., Al-Fayoumi S., Ligueros-Saylan M., et al.: J. Clin. Pharmacol. 50, 401 (2010).

12. Bloch M., Basile J.: J. Clin. Hypertens. 12, 809 (2010).

13. Ehara T., Irie O., Kosaka T., Kanazawa T, Breitenstein W, et al.: ACS Med. Chem. Lett. 5, 787 (2014). 
14. Aspiotis R., Chen A., Cauchon E., Dubé D., Falgueyret J.P., et al.: Bioorg. Med. Chem. Lett. 21, 2430 (2011).

15. Lacombe P., Arbour M., Aspiotis R., Cauchon E., Chen A., et al.: Bioorg. Med. Chem. Lett. 22, 1953 (2012).

16. Nakamura Y., Fujimoto T., Ogawa Y., Sugita C., Miyazaki S., et al.: ACS Med. Chem. Lett. 3, 754 (2012).

17. Nakamura Y., Sugita C., Meguro M., Miyazaki S., Tamaki K., et al.: Bioorg. Med. Chem. Lett. 22, 4561 (2012).

18. Tawada M., Suzuki S., Imaeda Y., Oki H., Snell G., et al.: Bioorg. Med. Chem. 24, 6066 (2016).

19. Winiecka I., Jaworski P., Mazurek A.P., Marszałek D., Goldnik A., Sokulski D.: J. Pept. Sci. 22, 106 (2016).

20. Paruszewski R., Jaworski P., Bodnar M., Dudkiewicz-Wilczyńska J., Roman I.: Chem. Pharm. Bull. 53, 1305 (2005).

21. Winiecka I., Dudkiewicz-Wilczyńska J., Roman I., Paruszewski R.: Acta Pol. Pharm. 67, 367 (2010).

22. Winiecka I., Marszałek D., Goldnik A., Jaworski P., Mazurek A.P.: Pharmazie 69, 263 (2014).

23. Winiecka I., Marszałek D., Goldnik A., Jaworski P., Mazurek A.P.: Acta Pol. Pharm. 71, 59 (2014).

24. Wu Y., Shi C., Sun X., Wu X., Sun H.: Bioorg. Med. Chem. 19, 4238 (2011).

25. Thaisrivongs S., Pals D.T., Harris D.W., Kati W.M., Turner S.R.: J. Med. Chem. 29, 2088 (1986).

26. Plattner J.J., Marcotte P.A., Kleinert H.D., Stein H.H., Greer J., et al.: J. Med. Chem. 31, 2277 (1988).

27. Kempf D.J., de Lara E., Stein H.H., Cohen J., Plattner J.J.: J. Med. Chem. 30, 1978 (1987).

28. Maibaum J., Rich D.H.: J. Org. Chem. 53, 869 (1988).
29. Iizuka K., Kamijo T., Harada H., Akahane H., Kubota T., et al.: Chem. Pharm. Bull. (Tokyo) 38, 2487 (1990).

30. Steulmann R., Klostermeyer H.: Liebigs Ann. Chem. 1975, 2245 (1975).

31. Schuda P.F., Greenlee W.J., Chakravarty P.K., Eskola P.: J. Org. Chem. 53, 713 (1988).

32. Schwyzer R., Sieber P., Kappeler H.: Helv. Chim. Acta 42, 2622 (1959).

33. Anderson G.W., McGregor A.C.: J. Am. Chem. Soc. 79, 6180 (1957).

34. Paruszewski R., Jaworski P., Tautt J., Dudkiewicz J.: Pharmazie 52, 206 (1997).

35. König W., Geiger R.: Chem. Ber. 103, 2034 (1970).

36. Marszałek D., Goldnik A., Mazurek A.P., Winiecka I., Jaworski P.: Acta Pol. Pharm. 71, 545 (2014).

37. Marszałek D., Goldnik A., Winiecka I., Jaworski P., Mazurek A.P.: Acta Pol Pharm. 74, 393 (2017).

38. Ghose A.K., Prichett A., Crippen G.M.: J. Comput. Chem. 9, 80 (1988).

39. Dassault Systèmes BIOVIA, Discovery Studio Modeling Environment, Release 2017, Dassault Systèmes, San Diego 2016.

40. Scheiper B., Matter H., Steinhagen H., Stilz U., Bocskei Z., et al.: Bioorg. Med. Chem. Lett. 20, 6268 (2010).

41. Politi A., Durdagi S., Moutevelis-Minakakis P., Kokotos G., Mavromoustakos T.: J. Mol. Graph. Model. 29, 425 (2010).

42. Rahuel J., Rasetti V., Maibaum J., Rüege H., Göschke R., et al.: Chem. Biol. 7, 493 (2000).

43. Matter H., Scheiper B., Steinhagen H., Bocskei Z., Fleury V., McCort G.: Bioorg. Med. Chem. Lett. 21, 5487 (2011). 